Submitted to ApJ

Preprint typeset using $\mathrm{LT}_{\mathrm{E}} \mathrm{X}$ style emulateapj v. 11/10/09

\title{
THE DEBRIS DISK OF VEGA: A STEADY-STATE COLLISIONAL CASCADE, NATURALLY
}

\author{
S. MÜLLER \\ Astrophysikalisches Institut und Universitätssternwarte, Friedrich-Schiller-Universität Jena, \\ Schillergäßchen 2-3, 07745 Jena, Germany \\ T. LÖHNE \\ Astrophysikalisches Institut und Universitätssternwarte, Friedrich-Schiller-Universität Jena, \\ Schillergäßchen 2-3, 07745 Jena, Germany \\ A. V. KRIVOV \\ Astrophysikalisches Institut und Universitätssternwarte, Friedrich-Schiller-Universität Jena, \\ Schillergäßchen 2-3, 07745 Jena, Germany \\ (Received 2009 September 7; Accepted 2009 November 30) \\ Submitted to ApJ
}

\begin{abstract}
It has been argued that the photometric data and images of the archetypical debris disk around Vega may be in contradiction with the standard, steady-state collisional scenario of the disk evolution. Here we perform physical modeling of the Vega disk "from the sources". We assume that dust is maintained by a "Kuiper belt" of parent planetesimals at $\sim 100 \mathrm{AU}$ and employ our collisional and radiative transfer codes to consistently model the size and radial distribution of the disk material and then thermal emission of dust. In doing so, we vary a broad set of parameters, including the stellar properties, the exact location, extension, and dynamical excitation of the planetesimal belt, chemical composition of solids, and the collisional prescription. We are able to reproduce the spectral energy distribution in the entire wavelength range from the near-infrared to millimeter, as well as the mid-IR and sub-millimeter radial brightness profiles of the Vega disk. Thus our results suggest that the Vega disk observations are compatible with a steady-state collisional dust production, and we put important constraints on the disk parameters and physical processes that sustain it. The total disk mass in $\lesssim 100 \mathrm{~km}$-sized bodies is estimated to be $\sim 10$ Earth masses. Provided that collisional cascade has been operating over much of the Vega age of $\sim 350 \mathrm{Myr}$, the disk must have lost a few Earth masses of solids during that time. We also demonstrate that using an intermediate luminosity of the star between the pole and the equator, as derived from its fast rotation, is required to reproduce the debris disk observations. Finally, we show that including cratering collisions into the model is mandatory.

Subject headings: planetary systems: formation - circumstellar matter - radiation mechanisms: thermal - stars: individuals: Vega
\end{abstract}

\section{INTRODUCTION}

Debris disks - disks of planetesimals and dust around mainsequence stars - are known to encircle a sizeable fraction of main-sequence (MS) stars. The observed dust is believed to derive from collisions between unobserved planetesimals that were neither used to make up planets nor ejected from the system by the time when the nebular gas was dispersed (see Wyatt 2008, for a review).

A standard scenario of a debris disk evolution (e.g. Thébault et al. 2003; Krivov et al. 2006; Strubbe \& Chiang 2006; Thébault \& Augereau 2007; Löhne et al. 2008; Krivov et al. 2008; Thébault \& Wu 2008) can be summarized as follows. There is a relatively narrow belt of planetesimals ("birth ring") in orbits with moderate eccentricities and inclinations, exemplified by the classical Kuiper belt in the solar system. The orbiting planetesimals in the birth ring undergo collisional cascade that grinds the solids down to dust. At smallest dust sizes, stellar radiation pressure effectively reduces the mass of the central star and quickly (on the dynamical timescale) sends the grains into more eccentric orbits, with their pericenters still residing within

sebastian@astro.uni-jena.de the birth ring while the apocenters are located outside the ring. As a result, the dust disk extends outward from the planetesimal belt. The smaller the grains, the more extended their "partial" disk. The tiniest dust grains, for which the radiation pressure effectively reduces the physical mass by half, are blown out of the system in hyperbolic orbits. The radiation pressure blowout of the smallest collisional debris represents the main mass loss channel in such a disk. A steady state implies a balance between the production of dust by the collisional cascade and its losses by radiation pressure blowout.

Such disks are usually referred to as collision-dominated, as opposed to systems that - at dust sizes - are transportdominated (e.g. Krivov et al. 2000). In the latter case, radial transport of dust material by various drag forces occurs on shorter timescales then collisions. Then, additional removal mechanisms may play a significant role. For example, Poynting-Robertson (P-R) drag can bring grains close to the star where they would sublimate or deliver them into the planetary region where they would be scattered by planets. To be transport-dominated, the system should either have an optical depth below current detection limits (Wyatt 2005a) or be subject to transport mechanisms other than P-R drag, such as 
strong stellar winds around late-type stars (Strubbe \& Chiang 2006; Augereau \& Beust 2006). Most of debris disks detected so far are thought to be collision-dominated. Accordingly, transport-dominated systems are not considered here.

The size segregation in collision-dominated disks described above means that at different wavelengths the same disk would look differently (see, e.g., Fig. 17 in Thébault \& Augereau 2007). Measurements at longer wavelengths (sub-mm) probe larger grains, because they are cooler, and thus trace the parent ring. Such observations may also reveal clumps, if for instance there is a planet just interior to the inner rim of the parent ring, and parent planetesimals and their debris are trapped in external resonances, similar to Plutinos in 3 : 2 resonance with Neptune (Wyatt 2006; Krivov et al. 2007). At shorter wavelengths (far-IR, mid-IR), smaller (warmer) grains are probed. Thus the same disk appears much larger. It may appear featureless, even if the parent ring is clumpy, because strong radiation pressure (Wyatt|2006) and non-negligible relative velocities (Krivov et al. 2007) would liberate such particles from resonant clumps. As a result, they would form an extended disk, as described above, regardless of whether their parent bodies are resonant or not.

The observed statistics of infrared (IR) luminosities of debris disks around AFGK stars, including their dependence on the stellar age and spectral class, is largely consistent with the steady-state collisional evolution scenario presented above (Wyatt et al. 2007b; Löhne et al. 2008). Furthermore, a detailed spectral energy distribution (SED) from far-IR to $\mathrm{mm}$ wavelengths, which was measured for a handful stars, can be reproduced with the models of a steady-state collisional cascade (Krivov et al. 2008). There are some exceptions, however (see, e.g., Wyatt 2008, and references therein). For instance, some A-type stars have fractional luminosities that are too high compared to what is expected from collisionally evolving Kuiper belt analogs. A few percent of FGK stars show excess emission shortward of $24 \mu \mathrm{m}$ that must come from "asteroidal" region inside $~ 10 \mathrm{AU}$, which in some cases is also too high to be compatible with the standard scenario (Wyatt et al. 2007a). Besides, incidences and properties of debris disks around M-type stars remain uncertain: the observations are scarce, and the physics of such disks may be different due to low stellar luminosity and strong stellar winds (e.g., Strubbe \& Chiang 2006; Augereau \& Beust 2006). Despite these caveats, the majority of the debris disks discovered so far appears compatible with the steady-state collisional scenario. However, the vast majority of debris disks are as yet unresolved, and the set of observables is typically limited to the fractional luminosity and a few photometric points in the IR. Many more constraints on the disk properties could be posed by resolved images, if these available, especially at more than one wavelength. There are currently a dozen of disks with such datasets. Accordingly, in this paper we undertake probably one of the first attempts to investigate, whether all available data on one particular debris disk star are compatible with a steady-state collisional scenario of dust production and evolution. We choose Vega.

Vega was the first MS star around which an IR excess over the photospheric flux, indicative of debris dust, was discovered (Aumann et al. 1984). It is therefore treated as an archetypical debris disk star; MS stars with IR excesses are often named "Vega-type stars". Fluxes at wavelengths from mid-IR to millimeter have been measured with IRAS (Aumann et al.1984; Walker \& Wolstencroft 1988), KAO (Harper et al. 1984; Harvey et al. 1984), and ISO
(Heinrichsen et al. 1998). As a result, its SED is known relatively well. The Vega disk has been resolved in sub-mm and mm with JCMT (Zuckerman \& Becklin 1993; Holland et al. 1998), OVRO (Koerner et al. 2001), IRAM (Chini et al. 1990; Wilner et al. 2002), and CSO (Marsh et al. 2006). These observations reveal a clumpy ring of large dust grains between about 80 and 120 AU, suggesting a Kuiper belt analog. Wyatt (2003) and Reche et al. (2008) naturally explain the ring structure with a resonant trapping of dust parent bodies by a presumed Neptune- to Saturn-mass planet during their outward migration in the past.

Su et al. (2005) resolved the Vega system by Spitzer/MIPS at $24,70,160 \mu \mathrm{m}$. They found a featureless, huge disk extending up to $\sim 800 \mathrm{AU}$. Although it came as a surprise at the time when this discovery was made, it is no longer astonishing now. As noted above, this is exactly what is expected: a Kuiper belt-sized, clumpy ring of large dust grains seen in the sub-mm and a much more extended disk of small grains, producing a smooth brightness distribution evident in the midto far-IR. However, by fitting these data, Su et al. (2005) deduced a mysterious overabundance of blowout grains of $\sim 1 \mu \mathrm{m}$ in radius. Under the assumption of a steady-state collisional disk evolution over the Vega age, this would imply that the disk must have lost $\sim 3$ Jupiter masses of material, which appeared unlikely. Accordingly, they suggested a recent major collisional event as a possible explanation. More exotic alternative scenarios proposed to explain such a large fraction of blowout grains include a close stellar encounter (Makarov et al. 2005) and a dynamical instability event similar to what caused the Late Heavy Bombardment in the solar system (Wyatt et al.2007a). However, Kenyon \& Bromley (2008), who modeled the Vega debris disk as an aftermath of icy planet formation with their hybrid multi-annulus coagulation code, find their model to be capable of reproducing the Spitzer fluxes, questioning the need in alternative scenarios.

We note that the excessive amount of grains in blowout orbits inferred by Su et al. (2005) uncovers another problem. A steady-state collisional evolution implies a certain size distribution of dust. Typically, the amount of blowout grains instantaneously present in the steady-state system is much less than the amount of slightly larger grains in loosely bound orbits around the star. This is because the dust production of the grains of adjacent sizes is comparable, but the lifetime of bound grains (due to collisions) is much longer than the lifetime of blowout grains (disk-crossing timescale). A jump in the size distribution around the blowout size is a robust prediction of all collisional cascade models, even those that do not assume a steady state (e.g. those that describe short-lived consequences of a major break-up event). Thus the amount of blowout grains reported by Su et al. (2005) would necessitate an unrealistically huge amount of larger grains in bound orbits. And this conclusion would hold not only in a steadystate scenario, but also all alternative scenarios listed above would face the same problem.

Beside the outer disk, the inner part of the system reveals another peculiarity. Pioneering interferometry observations with CHARA/FLUOR in the near-IR (Absil et al. 2006) have led to the discovery of a dust cloud just exterior of the sublimation zone, well inside 1 AU ("exozodi").

Just like the dust in the system, the central star turned out to be unusual, too. Peterson et al. (2006) and Aufdenberg et al. (2006) found Vega to be a rapid rotator, which makes stellar parameters functions of the stellar latitude. Table 1 summarizes the stellar parameters relevant for this study. It remains 
TABLE 1

STELLAR PARAMETERS

\begin{tabular}{lccc}
\hline & Equator & Pole & Note \\
\hline$R\left[R_{\odot}\right]$ & $2.873 \pm 0.026$ & $2.306 \pm 0.031$ & 1 \\
$T_{\text {eff }}[\mathrm{K}]$ & $7900_{-400}^{+500}$ & $10150 \pm 100$ & 2 \\
$L_{*}\left[L_{\odot}\right]$ & $28_{-6}^{+8}$ & $57 \pm 3$ & 3 \\
$\log \left(g\left[\mathrm{cms}^{-2}\right]\right)$ & $4.074 \pm 0.012$ & $3.589 \pm 0.056$ & 1 \\
\hline$M_{*}\left[M_{\odot}\right]$ & \multicolumn{2}{c}{$2.3 \pm 0.2$} & 2 \\
Age $[\mathrm{Myr}]$ & \multicolumn{2}{c}{350} \\
\hline
\end{tabular}

Notes: (1) From Peterson et al. (2006), (2) From Aufdenberg et al. (2006), (3) Luminosity at the equator and at the poles derived from the equatorial and polar values of the stellar radius $R$ and effective temperature $T_{\text {eff }}$ and from the average stellar luminosity of $37 \pm 3 L_{\odot}$ (Aufdenberg et al. 2006) through the Stefan-Boltzmann relation.

unclear whether unusual properties of the disk are somehow related to those of the star.

In this paper, we re-address the question of whether the available data are compatible with a steady-state collisional scenario of dust production and evolution. Instead of simply seeking dust distributions - e.g. in the form of power laws - that would provide the best fit to the observables, we employ an approach described by Krivov et al. (2008). In this approach, we assume a planetesimal belt with certain properties, evolve it with a collisional code to generate the dust portion of the debris disk, calculate the emission of that dust, and compare it to the observed emission. The best fit can be achieved by varying the parameters that describe the planetesimal belt (e.g. its location and mass), rather than parameters of the dust distribution as is commonly done.

In Sect. 2 we describe the data and their reduction and in Sect. 3 dynamical and thermal emission models used in this paper. Section 4 presents our reference ("first-guess") disk model and compares it with the available observational data. In Sect. 5] we analyze the influence of various model parameters on the observables. Section 6 contains a discussion and Sect.7lists our conclusions.

\section{OBSERVATIONAL DATA}

From the Spitzer archive, we extracted MIPS images of the Vega system at 24,70 , and $160 \mu \mathrm{m}$, using the Leopard software]. After applying standard basic corrections, the data were further processed to remove remaining constant backgrounds. Column averages were used to eliminate the socalled jail-bar artifacts caused by saturated sources on $24 \mu \mathrm{m}$ images.

For each image, the center of the source was then determined by minimizing first order moments. For the $160 \mu \mathrm{m}$ images, the pointing information was used for that purpose. After that, radial profiles were derived in an iterative process with two steps: (i) sampling the interpolated image at discretely binned distances around the source center with a subpixel resolution and (ii) integrating and subtracting the resulting profile pixel-wise from the original. Five such iterations were performed for each image. Finally, all profiles for each wavelength were combined, using their median.

At $24 \mu \mathrm{m}$, the data reduction is complicated by the fact that the central part of the images $\left(<4^{\prime \prime}\right.$ or $\left.<30 \mathrm{AU}\right)$ is saturated. Still, after subtracting the reference Point Spread Function (PSF), the outer part of the brightness profile can be found

\footnotetext{
http://ssc.spitzer.caltech.edu/mips/
}

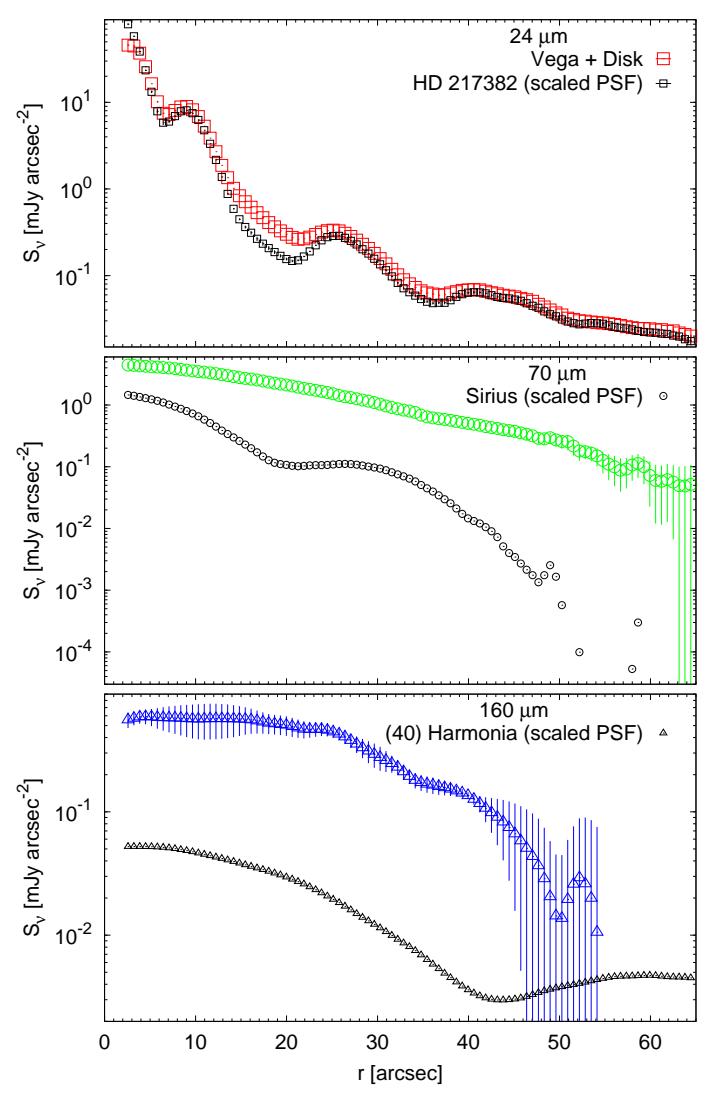

FIG. 1.- Radial profiles of the surface brightness for Vega as extracted from the Spitzer MIPS images for 24 (large squares), 70 (large circles), and $160 \mu \mathrm{m}$ (large triangles) from top to bottom. The standard sources HD 217382, Sirius, and (40) Harmonia used for PSF subtraction for the different wavelengths are shown as small symbols of the same shapes.

with sufficient accuracy. Figure 1 (top) shows the median profile extracted for Vega compared to that of the reference star HD 217382, a K4III giant at a distance of $120 \mathrm{AU}$, scaled up from its intrinsic $2.15 \mathrm{Jy}$ to the $7.4 \mathrm{Jy}$ (Su et al. 2005) of Vega's photosphere. The resulting photometric excess, obtained by integrating the subtracted profile (the Vega profile minus the scaled PSF) from 4 " outward, is $0.94 \pm 0.28 \mathrm{Jy}$. Here the formal error is determined by the standard deviations of Vega and the PSF plus an assumed systematic error of $5 \%$ in the photometry of Vega's photosphere. However, the flux is probably more uncertain because so is the contribution of the inner part of the disk, between 4" and 10" (30-80 AU). Thus, for a more accurate comparison with our models below, we can use the "certain" part of the observed flux by integrating the brightness profile from $10^{\prime \prime}$ outward. This nearly halves the total $24 \mu \mathrm{m}$ flux, giving $0.53 \mathrm{Jy}$. This "partial" flux will later be compared with the flux predicted by our models exactly in the same range of distances, from $10^{\prime \prime}$ (80 AU) outward. Coincidentally, it is this range where the emitting dust is only present in most of our models, because the inner edge of the birth ring is located at $80 \mathrm{AU}$. We emphasize, however, that the true total $24 \mu \mathrm{m}$ excess flux may be higher. It probably lies in the range $0.5-0.9 \mathrm{Jy}$, with no obvious possibility to narrow it because of the saturation problem described above.

The reduction of the $70 \mu \mathrm{m}$ images was more straightforward, resulting in the radial profiles shown in Fig. 11 (middle) and an excess of $8.6 \pm 1.2 \mathrm{Jy}$. Sirius (HD 48915) was used as reference.

Since stray light strongly contaminates images of hot, 
TABLE 2

PHOTOMETRIC DATA.

\begin{tabular}{rlll}
\hline$\lambda[\mu \mathrm{m}]$ & $F_{\text {disk }}[\mathrm{mJy}]$ & Instr. & Ref. \\
\hline 25.0 & $(2.979 \pm 0.936) \times 10^{3}$ & IRAS & Walker \& Wolstencroft (1988) \\
25.0 & $(4.019 \pm 2.080) \times 10^{3}$ & ISO & Heinrichsen et al. (1998) \\
47.0 & $(6.414 \pm 1.640) \times 10^{3}$ & KAO & Harvey et al. (1984) \\
60.0 & $(7.918 \pm 0.901) \times 10^{3}$ & IRAS & Walker \& Wolstencroft $(1988)$ \\
60.0 & $(9.318 \pm 2.082) \times 10^{3}$ & ISO & Heinrichsen et al. (1998) \\
80.0 & $(9.091 \pm 2.910) \times 10^{3}$ & ISO & Heinrichsen et al. (1998) \\
95.0 & $(6.829 \pm 1.825) \times 10^{3}$ & KAO & Harvey et al. (1984) \\
100.0 & $(7.109 \pm 0.754) \times 10^{3}$ & IRAS & Walker \& Wolstencroft $(1988)$ \\
100.0 & $(5.969 \pm 1.920) \times 10^{3}$ & ISO & Heinrichsen et al. (1998) \\
120.0 & $(4.890 \pm 1.551) \times 10^{3}$ & ISO & Heinrichsen et al. (1998) \\
170.0 & $(2.437 \pm 0.771) \times 10^{3}$ & ISO & Heinrichsen et al. (1998) \\
193.0 & $(8.932 \pm 5.000) \times 10^{2}$ & KAO & Harper et al. (1984) \\
200.0 & $(1.321 \pm 0.408) \times 10^{3}$ & ISO & Heinrichsen et al. (1998) \\
350.0 & $(4.691 \pm 1.500) \times 10^{2}$ & CSO & Marsh et al. (2006) \\
450.0 & $(1.301 \pm 0.450) \times 10^{2}$ & CSO & Marsh et al. (2006) \\
800.0 & $(1.626 \pm 0.500) \times 10^{1}$ & IRAM & Chini et al. (1990) \\
800.0 & $(2.426 \pm 1.500) \times 10^{1}$ & JCMT & Zuckerman \& Becklin $(1993)$ \\
850.0 & $(4.086 \pm 0.540) \times 10^{1}$ & JCMT & Holland et al. (1998) \\
870.0 & $(1.721 \pm 0.900) \times 10^{1}$ & IRAM & Chini et al. (1990) \\
1300.0 & $(2.390 \pm 1.500) \times 10^{0}$ & IRAM & Chini et al. (1990) \\
1300.0 & $(1.189 \pm 0.190) \times 10^{1}$ & OVRO & Koerner et al. (2001) \\
1300.0 & $(1.140 \pm 0.170) \times 10^{1}$ & IRAM & Wilner et al. (2002) \\
\hline & & & \\
& & & \\
& &
\end{tabular}

i.e. stellar, sources at $160 \mu \mathrm{m}$, we followed the strategy of Stapelfeldt et al. (2004) and used an additional reference star (HD 197989) to remove this artifact. However, visual inspection of the Vega images already suggested that the removal procedure would not be perfect because of some dissimilarities in the artifact shapes. Therefore, only the half-image facing away from the artifact center was used for profile extraction, as was done by Su et al. (2005). The actual reference used for PSF subtraction was the asteroid (40) Harmonia, although its influence on the excess of $1.9_{-0.5}^{+0.8} \mathrm{Jy}$ is negligible. See Fig.1 (bottom) for the resulting profiles.

The photometry points across the wavelength range from mid-IR to millimeter taken from the literature, are listed in Table 2. These points are plotted as symbols with error bars in bottom left panels of Figs. $5,13$.

\section{METHODS}

\subsection{Dynamical and Collisional Evolution}

Our technique to follow the size and radial distribution of material in rotationally-symmetric debris disks is described in detail in previous papers (Krivov et al. 2000, 2005, 2006, 2008; Löhne et al. 2008). Our numerical code, $A C E$ (Analysis of Collisional Evolution), solves the BoltzmannSmoluchowski kinetic equation over a grid of masses, periastron distances, and orbital eccentricities of solids. It includes the effects of stellar gravity, direct radiation pressure, drag forces, as well as disruptive and erosive collisions.

The results of $A C E$ simulations depend sensitively on the adopted collisional prescription. Possible collisional outcomes range from a perfect sticking or fragmentation with subsequent reaccumulation of particles (when the impact energy is low) to a crater formation or even a complete disruption (when it is high). An important quantity in the collisional prescription is the critical specific energy for disruption and dispersal, $Q_{\mathrm{D}}^{\star}$. It is defined as the impact energy per unit target mass that results in the largest remnant containing a half of the original target mass. For small objects, $Q_{\mathrm{D}}^{\star}$ is determined solely by the material strength, while for objects larger than $\sim 100 \mathrm{~m}$, the gravitational binding energy dominates. As a result, $Q_{\mathrm{D}}^{\star}$ is commonly described by the sum of two power laws (see, e.g., Davis et al.|1985; Holsapple 1994; Paolicchi et al. 1996; Durda \& Dermott 1997; Durda et al. 1998; Benz \& Asphaug 1999; Kenyon \& Bromley 2004b):

$$
Q_{\mathrm{D}}^{\star}=Q_{\mathrm{D}, \mathrm{s}}(s)\left(\frac{s}{1 \mathrm{~m}}\right)^{-3 b_{\mathrm{s}}}+Q_{\mathrm{D}, \mathrm{g}}(s)\left(\frac{s}{1 \mathrm{~km}}\right)^{3 b_{\mathrm{g}}},
$$

where the subscripts $s$ and g denote the strength and gravity regime, respectively.

The thermal emission in the mid- and far-IR is dominated by small dust with sizes slightly above the so-called blowout limit, below which particles are repelled from the system by stellar radiation pressure. An equilibrium size distribution set by the collisional cascade, especially near that blowout limit, depends on the total mass and the size distribution of fragments of each individual collision. Depending on the ratio of the impact energy to the critical energy, the average size of the fragments changes. This has been measured (e.g. Takagi et al. 1984) and numerically modeled (e.g. Durda et al. 2007), and useful analytic prescriptions for use in collisional codes are available (e.g. Thébault \& Augereau 2007). Here we apply a simplified approach with only one parameter, the slope $\eta$ of a single power law $\mathrm{d} N \propto m^{-\eta} \mathrm{d} m$. Larger values of $\eta$ put more weight on small fragments, while smaller values of $\eta$ "prefer" larger fragments. Note that using a single power law down to infinitely small grains is only meaningful for $\eta<2$.

Each $A C E$ run outputs, among other quantities, the size and radial distribution of disk solids over a broad size range from sub-micrometers to hundreds of kilometers at different time steps, and the code is fast enough to evolve the distribution over gigayears. As shown in Fig. 2, typical timescales for P$\mathrm{R}$ drag in the Vega disk are much longer than collisional lifetimes, except for a very narrow size range close to the blowout limit, where both become comparable. Thus we switch off the P-R effect in the $A C E$ runs, but make additional checks in Sect. 6.8 Gas drag can safely be neglected, because the Vega system with its 350 Myr age could not have retained any primordial gas, while the density of secondary gas cannot be high enough to influence the dust dynamics. As long as the drag forces are discarded, the number of parameters to vary, and thus the number of required $A C E$ runs, can be reduced by applying scaling laws derived in Löhne et al. (2008) and Krivov et al. (2008). Specifically, it is not needed to perform separate $A C E$ runs for the initial planetesimal disk with different initial masses.

\subsection{Thermal Emission of Dust}

Considering a rotationally symmetric disk of spherical particles, we denote by $N(r, s)$ the disk's surface number density of grains of radius $s$ at a distance $r$ from the star. The specific thermal emission flux from the entire disk $F_{\lambda \text {,disk }}^{\text {tot }}$ measured by an observer at a distance $D$ from the star at a given wavelength $\lambda$, can be calculated as (Krivov et al. 2008)

$$
\begin{aligned}
F_{\lambda, \text { disk }}^{\mathrm{tot}}= & \frac{2 \pi^{2}}{D^{2}} \int r\left(T_{g}\right) \frac{\mathrm{d} r\left(T_{g}\right)}{\mathrm{d} T_{g}} \mathrm{~d} T_{g} \int \mathrm{d} s s^{2} \times \\
& \times N(r, s) Q_{\lambda}^{a b s}(s) B_{\lambda}\left(T_{g}\right),
\end{aligned}
$$

where $B_{\lambda}\left(T_{\mathrm{g}}\right)$ is the Planck function, $Q_{\lambda}^{\mathrm{abs}}(s)$ is the absorption efficiency, $\lambda$ is the wavelength, and the grain temperatures $T_{\mathrm{g}}(s)$ are deduced from the standard assumption of thermal 


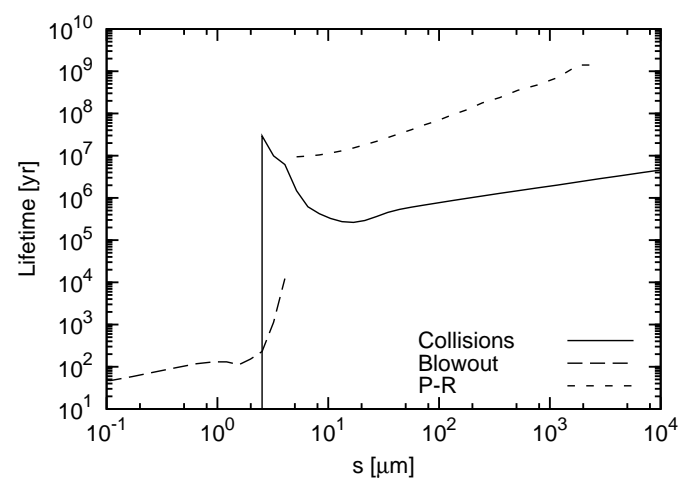

FIG. 2.- Timescales as functions of the grain size: collisional time (solid line), blowout time (long-dashed), and P-R time (short-dashed). The collisional time is an average over the grain orbits with all possible pericentric distances $q$ and eccentricities $e$, weighted with the amounts of those particles in the disk. The P-R time is the time it takes for a grain to drift across the parent ring or, more exactly, the time interval over which a grain's $q$ reduces from $120 \mathrm{AU}$ to $80 \mathrm{AU}$. It was computed by simultaneously solving the orbit-averaged equations that describe $q(t)$ and $e(t)$ (Burns et al. 1979). All timescales are for the parameters of the reference model (see Sect. 4).

equilibrium with the stellar radiation field. Similarly, the radial profile of the surface brightness $S_{\lambda}(r)$ is given by

$$
S_{\lambda}(r) \approx \frac{\pi}{D^{2}} \int \mathrm{d} s s^{2} N(r, s) Q_{\lambda}^{\mathrm{abs}}(s) B_{\lambda}\left(T_{\mathrm{g}}(r, s)\right) .
$$

To enable comparison with observations, equation (3) has to be convolved with the normalized PSF of the instrument:

$$
S_{\lambda}^{\prime}(\mathbf{r})=S_{\lambda}(\mathbf{r}) \otimes \operatorname{PSF}_{\lambda}(\mathbf{r}) .
$$

A numerical solution of Eqs. (2) and (3) has been implemented in two routines, SEDUCE (SED Utility for Circumstellar Environment) and SUBITO (SUrface Brightness Investigation TOol), respectively. The stellar flux needed to compute the dust temperatures is extracted from NextGen models (Hauschildt et al. 1999). Both codes have a direct interface to $A C E$, so that $A C E$ output can be used as input to SEDUCE and SUBITO. To arrive at the correct total dust mass and absolute values of fluxes, we rescale the ACE-SEDUCE and $A C E-S U B I T O$ simulation results with the aid of the appropriate scaling laws, as described in Appendix A of Krivov et al. (2008).

\section{THE REFERENCE MODEL OF THE VEGA DISK}

\subsection{Choice of Model Parameters}

In the reference model, we use the stellar luminosity at the equator of $L_{\star}=28 L_{\odot}$ (Table 1), and we assume that the collisional cascade has been operating over the entire stellar age, $350 \mathrm{Myr}$.

According to Dent et al. (2000), Su et al. (2005), Marsh et al. (2006) and Wyatt (2006) we adopt - as a "first guess" - an initial ring of parent bodies with semimajor axes ranging from 80 to $120 \mathrm{AU}$ and an initially constant surface density in this range. The clumpy shape of the sub-mm ring, usually interpreted through resonant capture of planetesimals by an unseen planet interior to the ring, implies that the eccentricities of the planetesimals are not very low (Wyatt 2003; Reche et al. 2008). On the other hand, the relatively narrow ring observed at wavelengths longer than $350 \mu \mathrm{m}$ indicates that eccentricities cannot be too high. As a reasonable compromise and for the sake of simplicity, for the reference model we adopt a uniform distribution of eccentricities from 0.0 to a moderate value of 0.2 . Maximum orbital inclinations (or a semi-opening angle) were then set to $0.1 \mathrm{rad}$ in accord with the energy equipartition assumption. Thus, the initial planetesimal disk resides between 64 and $144 \mathrm{AU}$ from the star. This is still in agreement with the observed 80 to $120 \mathrm{AU}$ as most of the material is concentrated in the central part of the initial ring. Note that these assumptions describe the initial disk extension. The subsequent collisional and dynamical evolution of the parent belt slightly changes the distributions of planetesimals.

All particles were assumed to be composed of astronomical silicate $\left(\rho=3.3 \mathrm{gcm}^{-3}\right.$, Laor \& Draine 1993). Mie theory was used to calculate radiation pressure and absorption efficiencies (Fig. 3).

The disk was modeled with $A C E$ with grains ranging from $0.05 \mu \mathrm{m}$ to $67 \mathrm{~km}$ in radius and the mass ratio in the adjacent bins of 4 . The pericenter distance grid covered 50 logarithmically-spaced values from $20 \mathrm{AU}$ to $800 \mathrm{AU}$. The eccentricity grid contained 100 linearly-spaced values between -5.0 and 5.0 (eccentricities are negative in the case of smallest grains with $\beta>1$, whose orbits are anomalous hyperbolas, open outward from the star). The distance grid used by $A C E$ to output distance-dependent quantities such as the size distribution was $10 \mathrm{AU}$ through $600 \mathrm{AU}$ at $10 \mathrm{AU}$ increments. In the collisional prescription, we set $Q_{\mathrm{D}, \mathrm{s}}(s)=$ $Q_{\mathrm{D}, \mathrm{g}}(s)=5 \times 10^{6} \mathrm{ergg}^{-1}, 3 b_{\mathrm{g}}=1.38$, and $3 b_{\mathrm{s}}=0.37$ and take the size distribution of fragments to be a power law with index $\eta=1.833$. Both disruptive and cratering collisions are switched on.

A set of parameters used for the reference model is given in the first line of Tab. 3. In the table, we only list those parameters that we later vary with respect to the reference model.

\subsection{Size, Radial, and Temperature Distributions}

Dust distributions in our reference model are presented in Fig. 4 The right panel shows the grain size distributions within and outside the birth ring. The radiation pressure blowout effect causes a steep drop between 3 and $5 \mu \mathrm{m}$, which corresponds to $\beta \approx 0.5$ (Fig. 3 right). As previous studies have shown, the blowout drop in the size distribution results in a more or less pronounced wavy pattern in the distribution of larger particles (e.g. Thébault et al. 2003; Krivov et al. 2006), with the "wavelength" and amplitude of the pattern depending on material strength and impact velocities. However, compared to previous studies (e.g. Krivov et al. 2006), $A C E$ now uses a grid of pericentric distances instead of semimajor axes. This reduces the effects of discretization on the effective relative velocities, which is strongest for particles on highly eccentric orbits with pericenters close to the birth ring. Therefore, the waviness is washed out, especially in the reference run.

The radial distribution of different-sized grains is shown in the bottom panel of Fig. 4 4 with thin lines. As explained above, most of the material is located between 80 and $120 \mathrm{AU} 2$. The largest particles are confined to this region as they are nearly unaffected by radiation pressure. The smaller the particles, the wider they are spread over the disk. In addition, the bottom panel in Fig. 4 plots the total normal geometrical optical

${ }^{2}$ Note that the ring starts at about $73 \mathrm{AU}$ and not at the above-mentioned $64 \mathrm{AU}$. This is due to the eccentricity binning. Individual bins are 0.1 wide and centered at $0.05,0.15$, and so on. Thus, the largest effective eccentricity for $e_{\max }=0.2$ is $e=0.15$. The corresponding minimum pericentric distance for $a=80 \mathrm{AU}$ is $q_{\min }=0.85 \times 80 \mathrm{AU}=68 \mathrm{AU}$. The next larger point in the pericenter grid is then centered at $73 \mathrm{AU}$. 

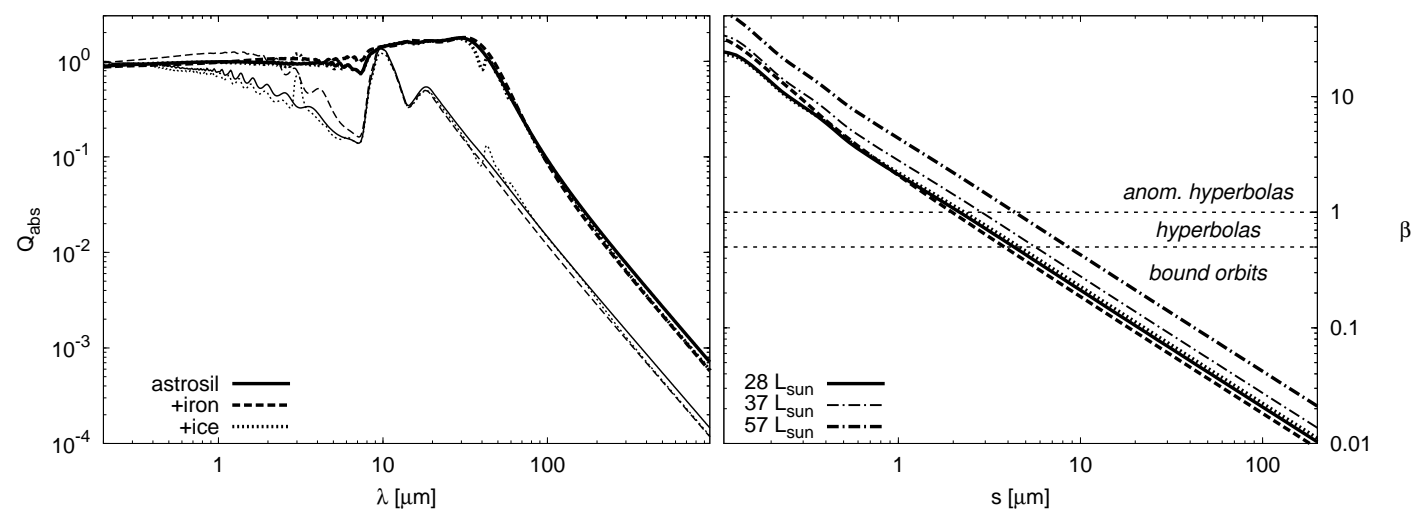

FIG. 3.- Left: Absorption efficiency for 1 (thin lines) and $4.9 \mu \mathrm{m}$ (thick lines) particles consisting of astronomical silicate (solid lines) and of an astronomic silicate matrix with $10 \%$ iron (dashed lines) and water ice (dotted lines) inclusions. Right: The $\beta$ ratio of the same grains as in the left panel (the same linestyles), assuming $L=28 L_{\odot}$. Additionally, thin and thick dash-dotted lines show the $\beta$ ratio for pure astrosilicate grains, but higher stellar luminosities of $L=37 L_{\odot}$ and $57 L_{\odot}$ respectively.

TABLE 3

SETS OF MODEL PARAMETERS USED IN THE SIMULATIONS. THE PARAMETERS OF THE REFERENCE MODEL ARE LISTED IN THE FIRST LINE. FOR ALL OTHER MODELS, ONLY PARAMETERS THAT ARE DIFFERENT FROM THOSE OF THE REFERENCE MODEL ARE GIVEN.

\begin{tabular}{cccccccccccc}
\hline Model & $L_{*}\left[L_{\odot}\right]$ & $T[\mathrm{Myr}]$ & $a_{\text {inner }}[\mathrm{AU}]$ & $a_{\text {outer }}[\mathrm{AU}]$ & $e_{\max }$ & $i_{\max }$ & composition & collisions & $Q_{D, s}\left[\mathrm{ergg}^{-1}\right]$ & $b_{s}$ & $\eta$ \\
\hline ref. & 28 & 350 & 80 & 120 & 0.2 & 0.1 & no incl. & w/ cratering & $5.0 \times 10^{6}$ & 0.37 & 1.833 \\
\hline a1 & - & 35 & - & - & - & - & - & - & - & - & - \\
a2 & - & 3.5 & - & - & - & - & - & - & - & - & - \\
b1 & - & - & 50 & - & - & - & - & - & - & - & - \\
b2 & - & - & 100 & - & - & - & - & - & - & - & - \\
b3 & - & - & - & 100 & - & - & - & - & - & - & - \\
b4 & - & - & - & 150 & - & - & - & - & - & - & - \\
c1 & 37 & - & - & - & - & - & - & - & - & - & - \\
c2 & 57 & - & - & - & - & - & - & - & - & - & - \\
c3 & - & - & - & - & - & - & ice incl. & - & - & - & - \\
c4 & - & - & - & - & - & - & iron incl. & - & - & - & - \\
d1 & - & - & 71.1 & 130.9 & 0.1 & 0.05 & - & - & - & - & - \\
d2 & - & - & 91.4 & 100.8 & 0.3 & 0.15 & - & - & - & - & - \\
e1 & - & - & - & - & - & - & - & w/o cratering & - & - & - \\
e2 & - & - & - & - & - & - & - & - & $2.5 \times 10^{6}$ & 0.2 & - \\
e3 & - & - & - & - & - & - & - & - & $6.9 \times 10^{6}$ & 0.45 & - \\
f1 & - & - & - & - & - & - & - & - & - & - & 1.6 \\
f2 & - & - & - & - & - & - & - & - & - & - & 1.95 \\
\hline fit & 45 & - & 62 & 120 & 0.1 & 0.05 & - & - & - & - & 1.95 \\
\hline
\end{tabular}

depth $\tau$ (in arbitrary scale). The optical depth beyond about $120 \mathrm{AU}$ is dominated by particles just above the blowout size, $\sim 5 \mu \mathrm{m}$, which are in barely bound orbits. It is only in the region of the birth ring where particles with $s \gtrsim 10 \mu \mathrm{m}$ make a significant contribution to the optical depth.

To judge about the dust temperatures and the thermal emission of the disk in the reference model, the left top panel in Fig. 4 depicts the dust temperature as a function of stellar distance and grain size. Both distance and size axes share those of the two other panels. Hence, extrapolating the maxima of the size and radial distributions into the temperature plot yields a "typical" temperature, i.e. the temperature of grains with $\tau$-dominating sizes at the distance where $\tau$ peaks. For our reference disk these are grains with $s \approx 5 \ldots 10 \mu \mathrm{m}$ at $r \sim 80 \ldots 120 \mathrm{AU}$ and their temperature is about $60 \mathrm{~K}$.

\subsection{Dust Mass and Disk Mass}

We then used SEDUCE to obtain the SED or the reference disk and to fit it vertically to the available observations starting at $25 \mu \mathrm{m}$. Shorter wavelengths were neglected in the fitting process as the uncertainty of the photospheric subtraction there is too high. Furthermore, we did not use the Spitzer $24 \mu \mathrm{m}$ data point for fitting because of the uncertainties in converting the images into photometry, as discussed in Sect. 2). In the SED calculation, we adopted the stellar parameters at the equator to obtain the photosphere seen by the dust disk, but took the polar values to calculate the observed photosphere (Tab. 1).

The aforementioned fitting itself is not as straightforward as it may seem. As we wish the modeled absolute thermal emission flux to match the actually observed one, we need to change the amount of dust, i.e. the dust mass. In our approach, however, only the parameters of the parent planetesimals can be changed, not those of dust they produce. It 


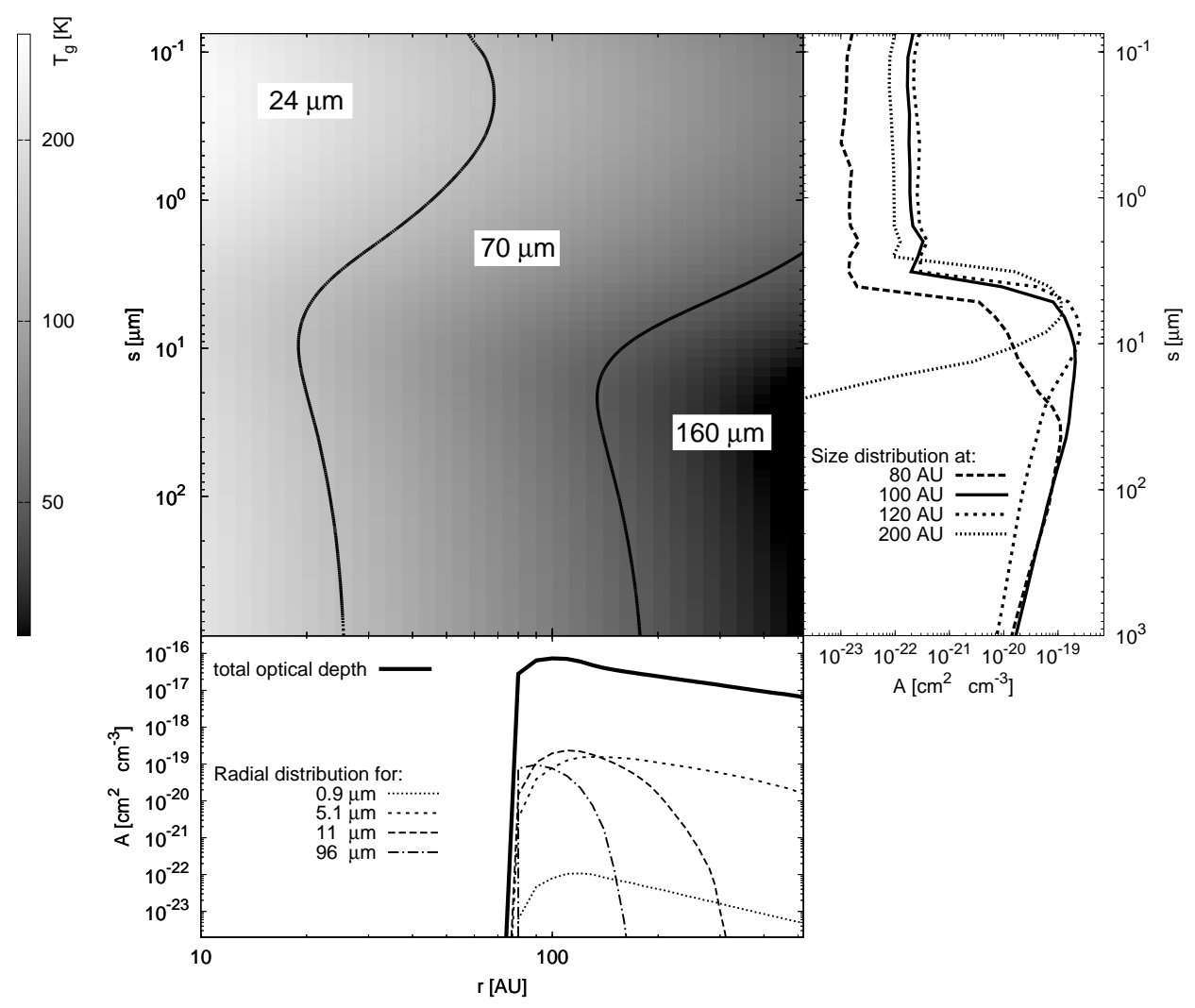

FIG. 4.- Left top: Disk's temperature profile for the assumed astrosilicate grains and stellar parameters as derived for Vega's equator. Two solid lines separate the regions of dominant emission at the three Spitzer/MIPS wavelengths: in the left part $24 \mu \mathrm{m}$ emission is more efficient than emission at the other two wavelengths, in the central part $70 \mu \mathrm{m}$ emission dominates, and in the right part $160 \mu \mathrm{m}$ emission is the strongest. Left bottom: Radial distribution in the reference model for $0.9,5.1,11$ and $96 \mu \mathrm{m}$ grains (thin lines) and the resulting total optical depth in arbitrary units (thick solid line). Right: Grain size distribution in the reference disk at $80,100,120$, and $200 \mathrm{AU}$.

means that we have to go one step back and modify the initial disk mass. However, it is not sufficient to change the initial disk mass by the ratio of the observed and the modeled fluxes. The reason is that a change in the initial mass also alters the rate of the collisional evolution, whereas we need the "right" flux at a fixed time instant, namely — in the reference model - at 350 Myr. Therefore, to find the mass modification factor we apply scaling rules, as explained in Appendix A of (Krivov et al. 2008).

The dust, disk, and initial disk masses in the reference model are given in the first line of Tab. 4 The dust mass of $7 \times 10^{-3} M_{\oplus}$ is by about a factor of two higher than what was derived by Su et al. (2006). The actual agreement is even better, because our upper cutoff size of $1000 \mu \mathrm{m}$ is larger than that of Su et al. The total mass of the reference disk is about $16 M_{\oplus}$, which is $85 \%$ of its initial mass $350 \mathrm{Myr}$ ago when the collisional cascade started to operate.

\subsection{Spectral Energy Distribution}

For an easier comparison between the modeled SEDs and the photometric observations in the different spectral regions, throughout the paper we use the excess ratio. The latter is defined as the ratio of the dust emission to the stellar photospheric emission or equivalently, as the ratio of the total flux (star + dust) to the stellar flux minus unity. The SED of the reference disk in terms of the excess ratio is presented in Fig. 5 (left bottom) with a solid line. Given that our reference model is a first-guess one, the agreement with the observations is quite satisfactory.

At $24 \mu \mathrm{m}$ the model yields $0.43 \mathrm{Jy}$, which is at $2 \sigma$ under
TABLE 4

DERIVED DUST MASSES, DISK MASSES, AND INITIAL DISK MASSES FOR ALL MODELS

\begin{tabular}{cccc}
\hline runs & $M_{\text {dust }}\left[10^{-3} M_{\oplus}\right]$ & $M_{\text {disk }}\left[M_{\oplus}\right]$ & $M_{\text {ini }}\left[M_{\oplus}\right]$ \\
\hline ref. & 6.63 & 16.3 & 18.9 \\
\hline a1 & 5.62 & 4.21 & 4.35 \\
a2 & 3.67 & 3.05 & 3.06 \\
b1 & 5.41 & 19.3 & 22.7 \\
b2 & 7.34 & 14.1 & 16.2 \\
b3 & 6.22 & 17.1 & 20.7 \\
b4 & 7.38 & 14.2 & 15.7 \\
c1 & 6.96 & 14.8 & 17.1 \\
c2 & 6.59 & 11.9 & 13.6 \\
c3 & 7.08 & 18.5 & 21.5 \\
c4 & 7.28 & 16.5 & 19.1 \\
d1 & 10.9 & 41.5 & 47.5 \\
d2 & 4.74 & 10.5 & 12.6 \\
e1 & 4.37 & 3.60 & 3.81 \\
e2 & 8.64 & 51.0 & 62.7 \\
e3 & 4.77 & 5.15 & 5.70 \\
f1 & 7.09 & 9.31 & 10.5 \\
f2 & 5.92 & 32.2 & 39.4 \\
\hline fit & 5.09 & 46.7 & 55.5 \\
\hline & & &
\end{tabular}




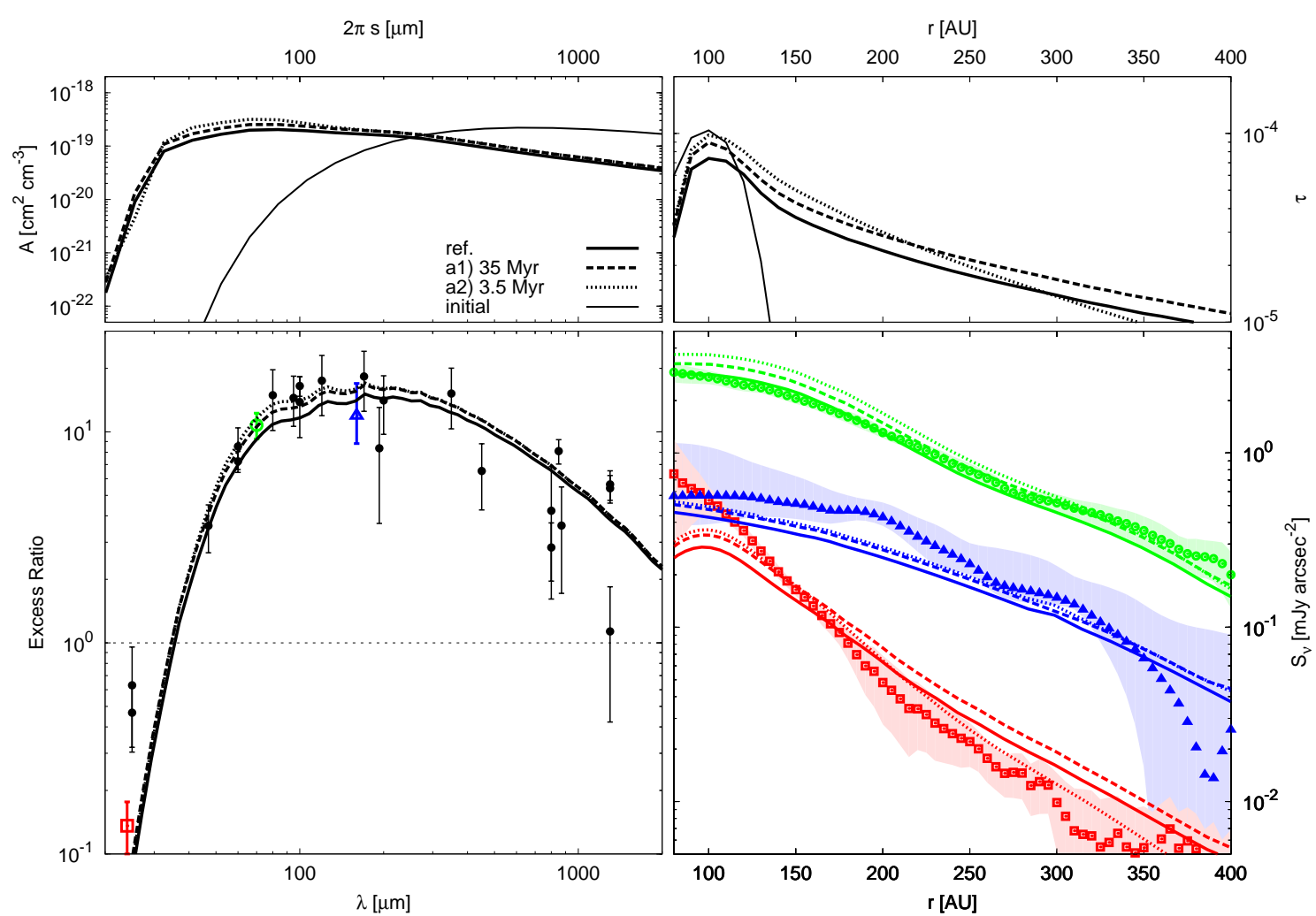

FIG. 5.- Top left: Grain size dust distributions of the reference model (thick solid line) and of the same model but at earlier times of 3.5 (dotted line) and $35 \mathrm{Myr}$ (dashed line), all in the center of the initial planetesimal ring. The initial distribution for the simulation is plotted as thin solid line. Note our using $2 \pi s$ instead of $s$ in this and subsequent size distribution plots. Since particles with the size parameter $2 \pi s / \lambda \sim 1$ emit most efficiently, $2 \pi s$ roughly gives the typical wavelength of the emission. This alleviates comparison between the size distribution and the SED (bottom left). Top right: Radial profile of the optical depth for the same disk models. Bottom left: Corresponding SEDs. Symbols with error bars are data points (large square, large circle, and large triangle mark $24 \mu \mathrm{m}$, $70 \mu \mathrm{m}$, and $160 \mu \mathrm{m}$ excess ratios deduced from Spitzer/MIPS images. Bottom right: Modeled (lines) and observed (symbols) surface brightness profiles at 24 , 70 , and $160 \mu \mathrm{m}$. The shapes of symbols are the same as in the bottom left panel and in Fig. 10 The shaded areas around the data points indicate the errors.

the Spitzer point of 0.94 Jy. Let us make a more careful comparison, however. As explained in Sect. 2, the total observed flux from $4^{\prime \prime}$ outward is unsure, because of an uncertain part between $4^{\prime \prime}$ and $10^{\prime \prime}$. If we consider only the observed flux from $10^{\prime \prime}$ outward, it reduces to $0.53 \mathrm{Jy}$. At the same time, we can calculate the flux from $10^{\prime \prime}$ outward in the reference model. The result is $0.40 \mathrm{Jy}$, which is only slightly below the observed one. That value of $0.40 \mathrm{Jy}$ differs insignificantly from the calculated flux from 4 " outward, $0.43 \mathrm{Jy}$, because the emitting dust in the reference model is entirely located outside $10^{\prime}$ ", and it is only the finite PSF's width that "transfers" 0.03 Jy of the emission closer in.

As far as the IRAS $25 \mu \mathrm{m}$ flux is concerned, it is known to be quite uncertain due to the large field of view and it is inconsistent with the Spitzer $24 \mu \mathrm{m}$ measurement anyway.

In the far-IR the model emission matches the Spitzer fluxes perfectly and lies within the error bars of the other observations. At (sub-)millimeter wavelengths, the measurements themselves are contradictory, lying sometimes at more than $2 \sigma$ from each other, and it is difficult to judge which of them are most accurate. The model SED provides a compromise, lying in the middle of the entire set of data points.

\subsection{Radial Surface Brightness Profiles}

Using SUBITO, we calculated the radial surface brightness profiles of our disk model and convolved them with the corresponding PSFs. The final profiles are presented as solid lines in the right panel of Fig. 5. The model profiles are not inconsistent with the Spitzer observations. Especially the $70 \mu \mathrm{m}$ profile is very close to what was measured. However, both 24 and $160 \mu \mathrm{m}$ curves are slightly too flat. The $160 \mu \mathrm{m}$ profile lies under the measurements in the inner part of the disk and above them in the outer part, explaining why the total $160 \mu \mathrm{m}$ flux is about right (see the bottom left panel). In contrast, most of the $24 \mu \mathrm{m}$ flux comes from the inner part of the disk inside $100 \mathrm{AU}$. In this region the model profile goes below the data points, so that the higher emission in the outer part of the disk cannot compensate this deficiency.

Altogether, we state that the surface brightness profiles are more constraining for the disk model than the SED. Already our "first-guess" model satisfactorily reproduces the observed SED, but the brightness profiles reveal moderate deviations from those deduced from the observations.

\section{VARIATION OF MODEL PARAMETERS}

In this section we investigate how the observables (SED, brightness profiles) respond to changes in physical parameters (those of the star, planetesimal belts, and dust, as well as the collisional prescription). A specific goal is to check if we can improve the agreement of the modeled brightness profiles of the Vega disk with observations, preserving the agreement in the SED that we achieved in the reference model. Accordingly, we consider a set of models, the parameters of which are listed in Tab. 3. Most of these models differ from the reference model by one parameter. We modify several parameters at a time only if these are physically related and this is required for consistency.

For each of the models, we present the results in the same 
way as for the reference one. The size distribution, optical depth profile, the SED, and the radial brightness profiles are combined into a single figure (Figs. 6 to 13), each having the same structure as Fig. 5] In all the figures, the reference model is overplotted with a solid line. The resulting final dust masses, final disk masses, and initial disk masses are given in Tab. 4.

In the following subsections the variations of the reference model are explained and discussed. They are structured according to the underlying physical and astrophysical mechanisms at work.

\subsection{Delayed Stirring}

Before a debris disk starts to evolve in a steady-state regime, a collisional cascade has to ignite and operate for sufficient time. Initiation of the cascade requires a mechanism to stir the disk (Wyatt 2008). This can be self-stirring by largest planetesimals (Kenyon \& Bromley 2004a) or stirring by planets orbiting in the inner gap of the disk (Wyatt 2005b; Mustill \& Wyatt 2009). External events such as stellar flybys can also stir the disk sufficiently, it may have been the case for Vega $\sim 5 \mathrm{Myr}$ ago (Makarov et al. 2005). Furthermore, after the onset of the cascade, the system needs enough time to reach a steady-state collisional regime at dust sizes (e.g. Löhne et al. 2008). Thus the duration of a steady-state disk evolution is generally shorter than the system's age. We do not know which particular mechanism may have triggered the cascade in the Vega disk and how long it is already at work. It may have started either shortly after the primordial gas dispersal or much later in the Vega history.

To investigate the effect of the unknown "collisional age" of the Vega disk, we simply took our reference disk model and calculated the SEDs and surface brightness profiles at earlier time steps. Fig. [5 shows the results at 3.5, 35 and $350 \mathrm{Myr}$. At earlier times, the maximum of the size distribution is slightly more pronounced (top left). This results in a moderate enhancement of thermal emission between 50 and $500 \mu \mathrm{m}$, which is still in agreement with the observations (bottom left). The optical depth profile (top right) shows that 3.5 Myr of collisional evolution is not sufficient to bring enough particles on highly eccentric orbits, so that the profile is steeper than in the reference model. The $24 \mu \mathrm{m}$ and $70 \mu \mathrm{m}$ profiles steepen (bottom right), the latter being no longer compatible with observations. At $35 \mathrm{Myr}$, the optical depth profile is only shifted vertically compared to the reference model, which indicates that the spatial distribution has already reached an equilibrium (top right). The final and initial disk masses (Tab.4) are close to each other, which is natural as younger disks have spent less material in collisions. Besides, the estimated total masses of younger disks are smaller than in the reference model. The reason is that younger disks that are not in a steady-state regime yet are "dustier" than older disks of the same total mass (Krivov et al. 2006).

An overall conclusion is that at least several tens of Myr of collisional evolution seem to be required to make observables consistent with observations.

\subsection{Disk Location}

Our choice of the initial disk extension in the reference model comes from resolved images in the sub-mm and radio. However, a low resolution of these observations still leaves room for reasonable modifications. Hence we now vary the initial semimajor axis range of planetesimals, intentionally pushing them to the limits posed by the images, in order to see the effects more clearly.

We start with the inner disk edge. By placing additional material closer in, one may expect to increase the warm emission and prevent the brightness profile from dropping off towards the star too early. Thus, we try shifting the inner edge in to $50 \mathrm{AU}$. For completeness, we also add the case with the inner edge at $100 \mathrm{AU}$. The results are presented in Fig. 6.

As expected, taking $a_{\text {inner }}=50 \mathrm{AU}$ slightly shifts the SED to shorter wavelengths and strongly depresses the sub-mm emission. Taking $a_{\text {inner }}=100$ AU yields the opposite, although the effect is weaker. This traces back to the under- or overabundance of larger grains, hundreds of micrometers in size (top left). The reason for that, in turn, is the existence of two distinct dynamical regimes for bound dust grains. Large grains with no or little response to the stellar radiation pressure essentially inherit their orbital eccentricities from the parent bodies. For small, barely bound grains, radiation pressure is the dominant effect, pushing them to wide, highly eccentric orbits. Shifting the disk further in increases the average collisional velocities, lowers the collisional lifetime of larger grains - but not of smaller ones. As a result, the relative abundance of larger grains is reduced.

In terms of the surface brightness profiles, it is mostly the $24 \mu \mathrm{m}$ profile that is affected. It rises significantly inside $200 \mathrm{AU}$, reproducing perfectly the observations. However, in the outer part of the disk it becomes flatter so that the emission here is overestimated. The $70 \mu \mathrm{m}$ profile remains almost unaffected, except for the inner part within $150 \mathrm{AU}$, which responds to $a_{\text {inner }}$ in the same manner the $24 \mu \mathrm{m}$ does, albeit less strongly. Finally, the $160 \mu \mathrm{m}$ profile preserves its slope, but shifts downwards $\left(a_{\text {inner }}=50 \mathrm{AU}\right)$ or slightly upwards $\left(a_{\text {inner }}=100 \mathrm{AU}\right)$.

Similar to the inner edge, an inward shift of the outer edge would lower the amount of cold dust, enhancing the warm emission. So we changed $a_{\text {outer }}$ to 100 and $150 \mathrm{AU}$ to find similar modifications in the dust distribution and thermal emission as above (Fig. 7). Decreasing $a_{\text {outer }}$ makes the ring narrower and shifts the bulk of the material closer in. The maximum in the size distribution becomes more pronounced and shifts to smaller grains. The entire SED slightly shifts towards shorter wavelengths. The peak of the optical depth profile becomes stronger and moves closer to the star. This directly translates to the radial surface brightness profiles, especially at $24 \mu \mathrm{m}$, which becomes appreciably steeper. Increasing $a_{\text {outer }}$ naturally has opposite effects.

On the whole, it seems that shifting the inner edge of the belt inward has a clear potential of getting the $24 \mu \mathrm{m}$ profile that would better match the observed one. However, the shift from $80 \mathrm{AU}$ down to $50 \mathrm{AU}$ that we have tested may be too strong, because it may contradict to the sub-mm images.

\subsection{Dynamical Excitation}

We now consider the dynamical excitation of the disk, as parameterized by the maximum orbital eccentricities $e_{\max }$ that planetesimals had at the onset of the collisional cascade. This value does not change considerably in the course of the subsequent evolution (under the assumptions of our model, e.g. without planets), and it is approximately the same for disk solids of all sizes except for the smallest dust particles that are vulnerable to radiation pressure. From the dynamical point of view, higher eccentricities increase the collisional velocities (although the collisional rates remain nearly the same, see, e.g. Oueck et al 2007) and thus the efficiency of the colli- 


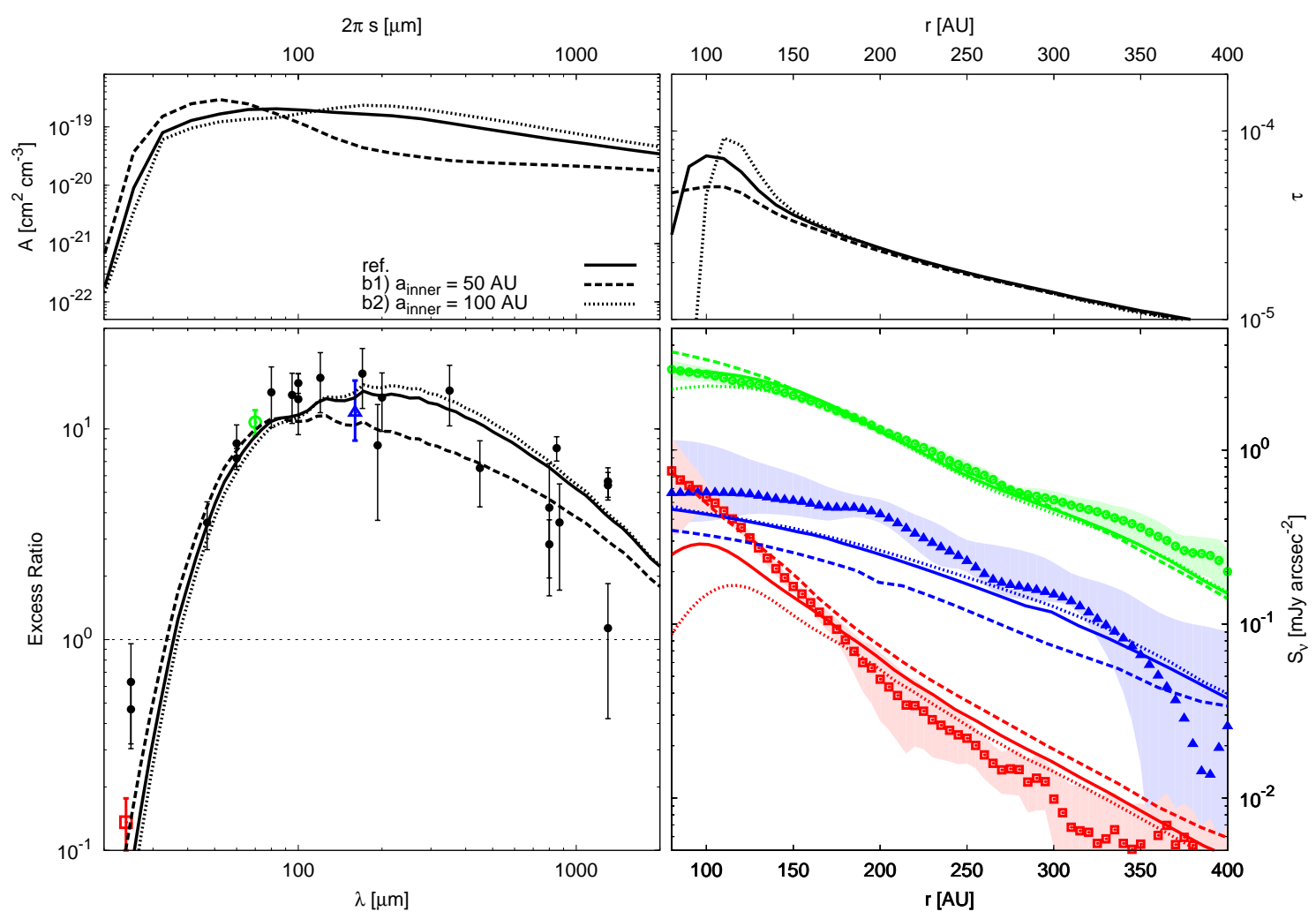

FIG. 6. - Same as Fig. 5 but with the inner edge of the disk shifted inward (dashed lines) and outward (dotted).

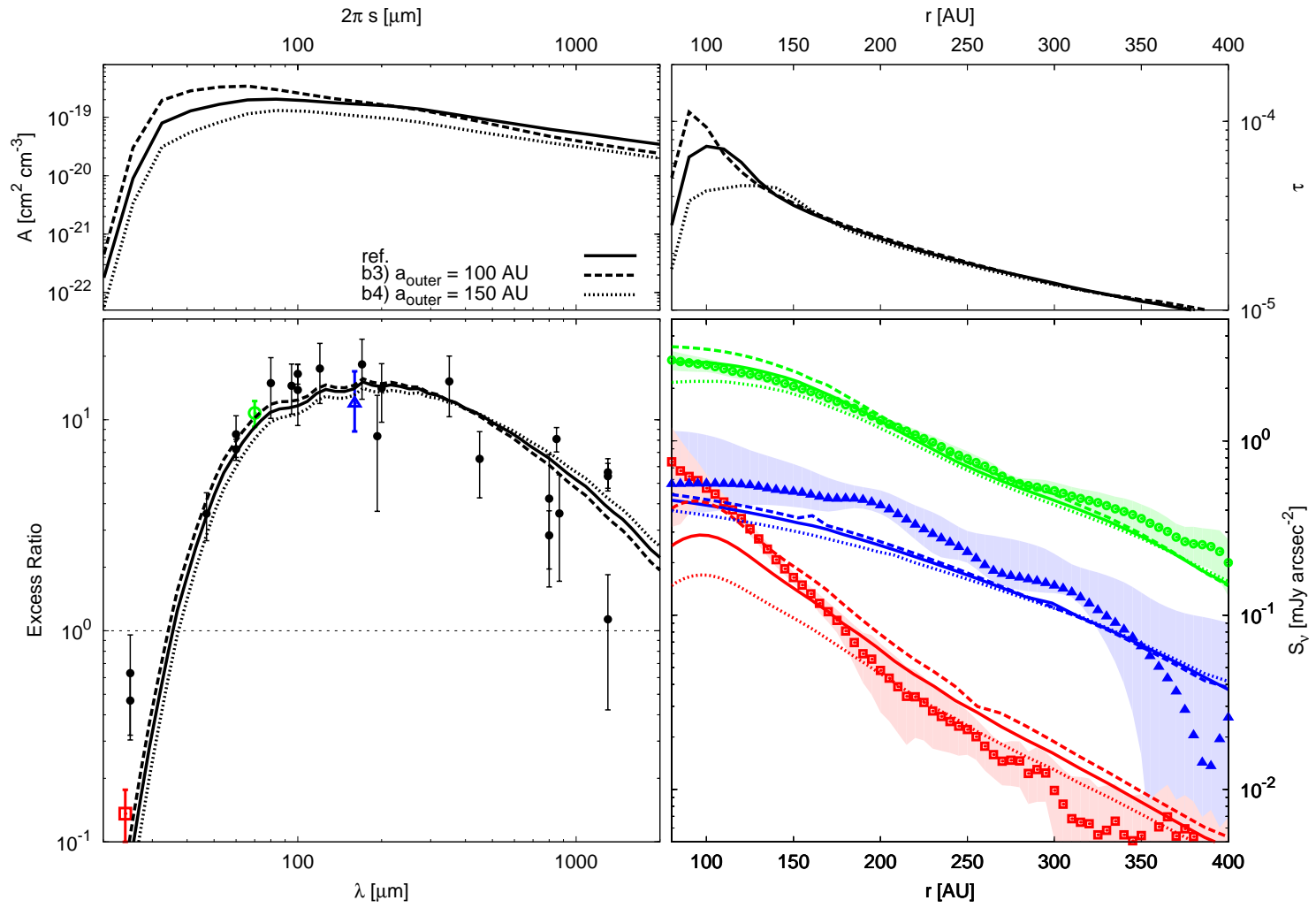

FIG. 7. - Same as Fig. 5 but with the outer edge of the disk shifted inward (dashed lines) and outward (dotted). 
sional cascade.

We ran two simulations: one with reduced $\left(e_{\max }=0.1\right)$ and one with increased eccentricity $\left(e_{\max }=0.3\right)$. The inclination of the disk was taken to fulfill the energy equipartition condition, $i_{\max }=e_{\max } / 2$. Since changing $e_{\max }$, but maintaining the same distribution of semimajor axes would change the radial extension of the disk, we chose to alter $a_{\text {inner }}$ and $a_{\text {outer }}$ in such a way as to preserve the radial extension of the reference model disk. (Strictly speaking, all this applies to the initial disk, because we have no control over the disk extension at later times in the course of its dynamical evolution.)

The size distribution in Fig. 8 shows that higher eccentricities lead to a depletion of larger grains $(>30 \mu \mathrm{m})$ and in return to an overabundance of smaller grains close to the blowout size. Consequently, the SED drops beyond $\approx 200 \mu \mathrm{m}$ and rises at shorter wavelengths becoming more narrow. In contrast, with lower eccentricities more grains of radii $>100 \mu \mathrm{m}$ survive, so that fewer particles with $15 \mu \mathrm{m}<s<100 \mu \mathrm{m}$ are created in collisions, which results in a more pronounced maximum between the blowout and $30 \mu \mathrm{m}$. This yields an enhancement of the radio emission and an overall flatter shape of the SED.

The slope of the optical depth profile in the outer part of the disk remains nearly unchanged, it just shifts vertically. However, in the range of the birth ring the optical depth profiles for different $e_{\max }$ are different: the lower $e_{\max }$, the broader the maximum. It is because for higher $e_{\max }$, the distribution of semimajor axes is narrower and the collisional production of dust near the center of the ring is much higher than elsewhere. For lower $e_{\max }$, the semimajor axes are distributed more broadly and dust is collisionally produced at comparable rates everywhere in the ring.

The brightness profiles respond to the changes in the disk excitation in a similar way. In the outer part they just shift vertically, and most of the changes are in the birth ring region. When $e_{\max }$ is reduced to 0.1 , in the outer disk the $24 \mu \mathrm{m}$ profile matches the observed profile closely. In the region of the birth ring it is still too low, as is the reference profile. However, the emission now keeps rising inward down to $80 \mathrm{AU}$, i.e. all the way through the birth ring, as does the observed emission.

\subsection{Stellar Luminosity}

Changing the stellar luminosity has a two-fold effect on the results. First, it alters the $\beta$-ratio of the dust grains, affecting their dynamics. Second, a different luminosity affects the temperature of the dust grains, thereby changing the SED and brightness profiles. Note that all these changes influence only the dust portion of the disk, not the larger objects.

As mentioned above, Vega is a rapid rotator and so the radiation flux emitted from its surface varies with the stellar latitude. In the reference model, we adopted the "equatorial luminosity", $28 L_{\odot}$. However, the dust disk "sees" not only the stellar equator, but also receives stellar radiation from higher altitudes. Thus, we now test the average luminosity of $37 L_{\odot}$ as derived by Aufdenberg et al. (2006) and, as an extreme case, the canonical polar value of $57 L_{\odot}$ (used by many modelers before).

As the luminosity gets higher, the blowout size increases (Fig. 3, right), reaching $10 \mu \mathrm{m}$ for a $57 L_{\odot}$ central star. The entire size distribution shifts horizontally towards larger sizes and the jump at the blowout radius becomes more abrupt (Fig. 9). The optical depth profile preserves its shape, but moves downward. The reason for the decrease of the optical depth level at higher luminosities is simply the increase of the grains' blowout size, so that there are fewer grains that could stay in bound orbits.

Interestingly, the SED in Fig. 9 (bottom left) does not change substantially, as one would expect from dramatic changes in the size distribution. The reason for moderate changes seen in the figure can be found by analyzing the size distribution (Fig. 4, top right) and the temperature plot (Fig. 4 top left). Since for a larger $L$ the size distribution is shifted to larger particles, the temperature range of the smallest particles, which affects the outer part of the disk the most, becomes narrower. Consequently, the SED slightly narrows, too, and the maximum at $\sim 100 \mu \mathrm{m}$ becomes more pronounced.

The changes in the surface brightness profiles are two-fold. While the $24 \mu \mathrm{m}$ profile steepens with increasing luminosity, the 160 and especially the $70 \mu \mathrm{m}$ curves flatten. The explanation for this behavior is as follows. The farther out from the star, the faster the temperature decreases with increasing grain size (Fig. 4, top left). A comparison with the position of the maxima in the size distribution at different distances and for different luminosities (Fig. 4 top right) demonstrates that an average temperature in the region of the birth ring increases with increasing $L$. In the outer disk the effect is reverse: far from the star, a higher stellar luminosity lowers the typical dust temperatures. These effects explain why for higher luminosities the mid-IR emission rises in the inner disk and drops in the outer one, steepening the $24 \mu \mathrm{m}$ profile. At the same time, the far-IR emission becomes more efficient farther out, which flattens the 70 and $160 \mu \mathrm{m}$ profiles.

Our analysis definitely favors an intermediate value of the Vega luminosity, exemplified by $L=37 L_{\odot}$ in our tests. First, this choice is well justified physically. Indeed, dust is exposed to stellar light coming from a range of latitudes, thus the "right" luminosity should be between the equatorial and polar one. Second, it does provide a better agreement with observations. Changes in the 70 and $160 \mu \mathrm{m}$ profiles are only marginal, so that they still match the observations well enough, while the $24 \mu \mathrm{m}$ profile steepens inside $\approx 250 \mathrm{AU}$, coming much closer to the observed profiles.

\subsection{Chemical Composition}

Like the stellar luminosity, the chemical composition of grains affects both the $\beta$-ratio (through the radiation pressure efficiency and bulk density) and dust temperatures (through the absorption efficiency).

Mid- and far-IR spectra of some debris disks reveal distinctive features (Jura et al. 2004; Chen et al. 2006), which allows one to get insight into the mineralogy of the dust grains. For example, spectra of several disks were matched by a mixture of amorphous and crystalline silicates, silica, and several other species (Schütz et al. 2005; Beichman et al. 2005; Lisse et al. 2007, 2008), including possibly water ice (Chen et al.2008). Unfortunately, the spectra of the Vega disk (available in the Spitzer archive) do not exhibit unambiguous features, which poses no observational constraints on its composition.

In the reference model we used pure astronomical silicate. Now, to test possible effects of chemical composition, we consider an astrosilicate matrix with water ice (Warren 1984) and iron (Lynch \& Hunter 1991) inclusions. The refractive indices were calculated according to the Maxwell-Garnett theory. The amount of inclusions was limited to $10 \%$, which is an upper limit for which the effective medium theory still provides accurate results (Kolokolova \& Gustafson 2001). The resulting bulk densities for the mixtures with ice and iron 


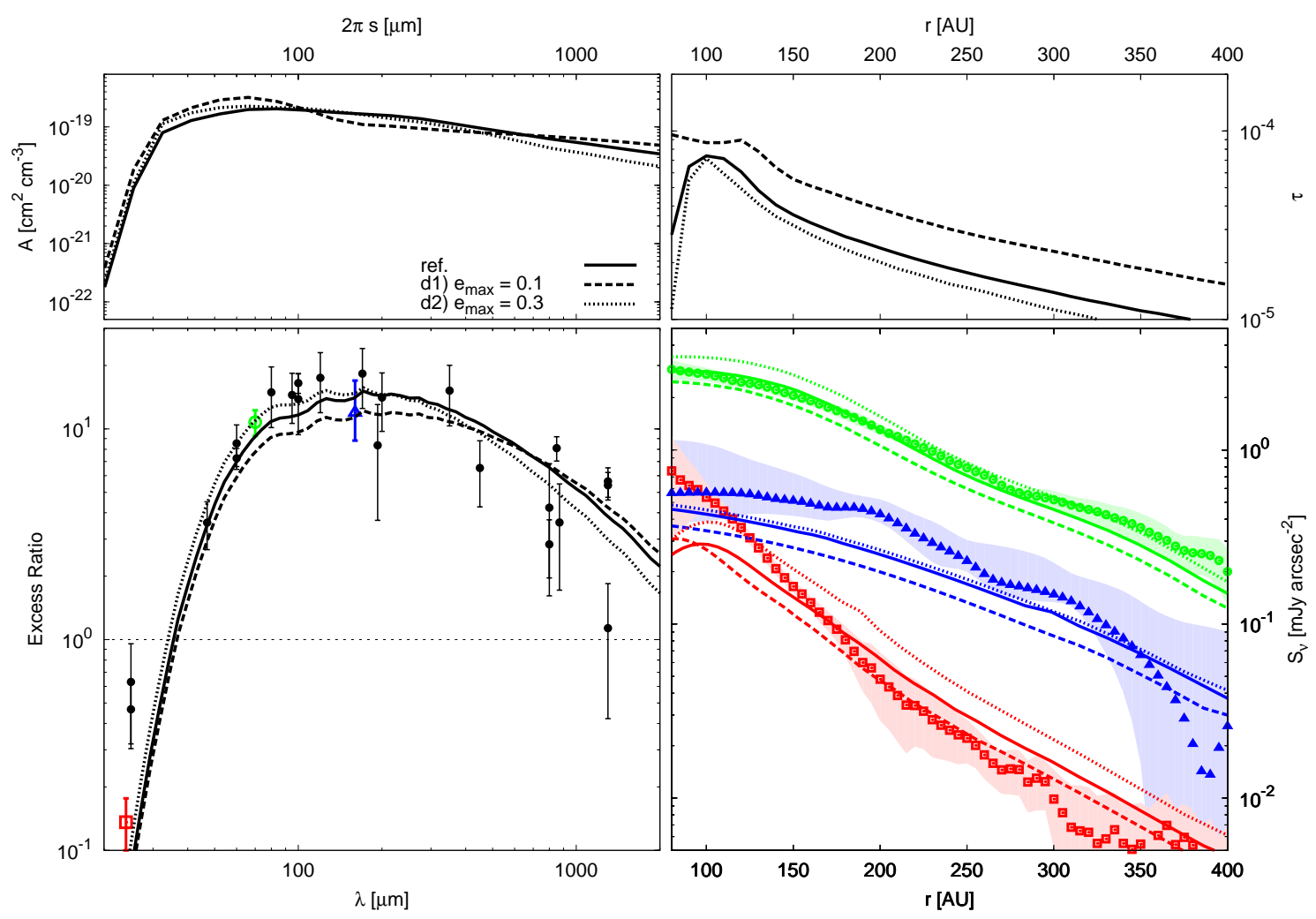

FIG. 8.- Same as Fig. [5 but for dynamically less (dashed lines) and more (dotted lines) excited model disks.

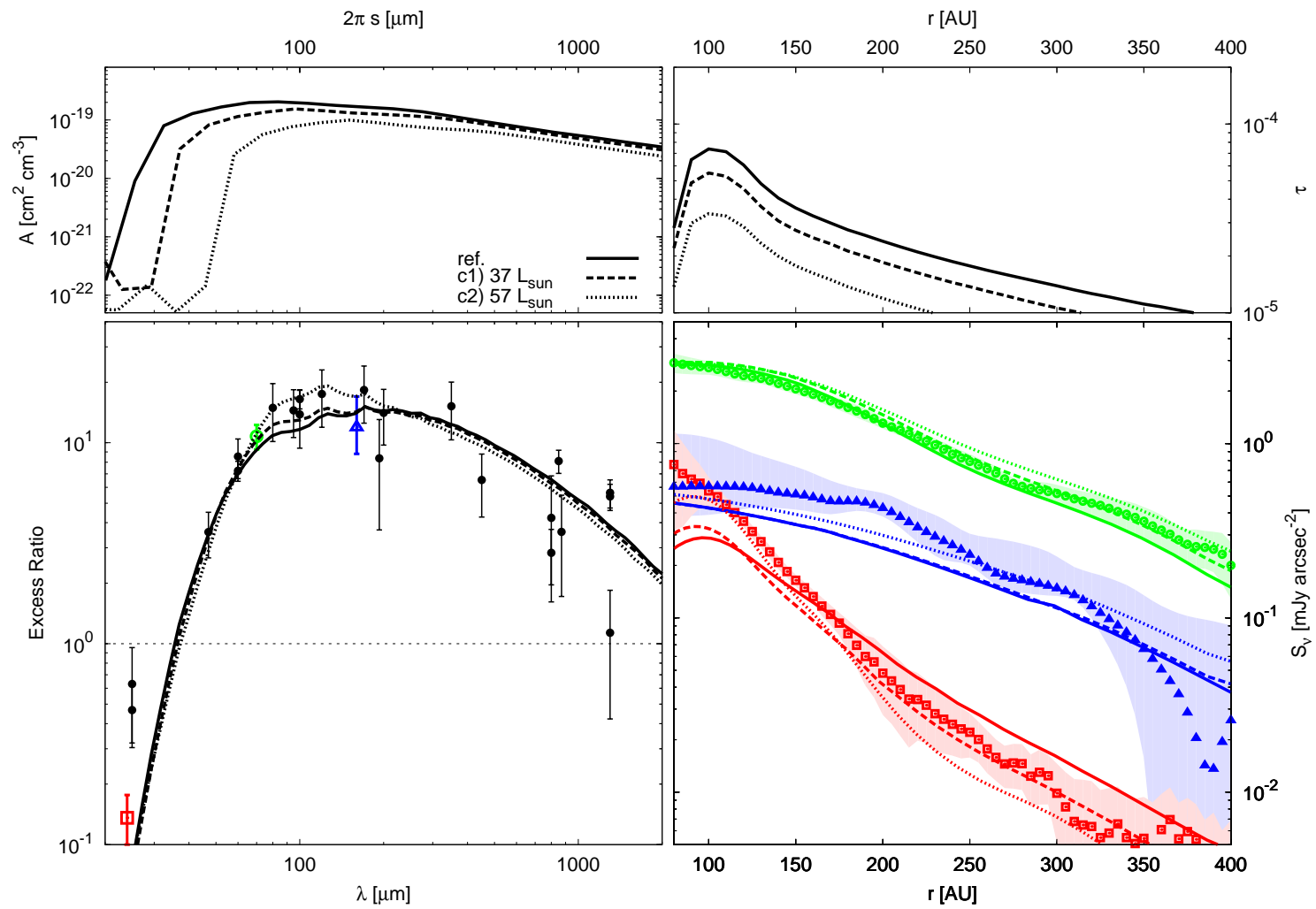

FIG. 9.- Same as Fig. 5 but assuming a more luminous central star with luminosity of 37 (dashed lines) and $57 L_{\odot}$ (dotted lines). 
are $3.062 \mathrm{~g} / \mathrm{cm}^{3}$ and $3.757 \mathrm{~g} / \mathrm{cm}^{3}$, respectively (compared to $3.3 \mathrm{~g} / \mathrm{cm}^{3}$ for pure astrosilicate). The effect of inclusions on the absorption efficiencies (except for small grains in the nearIR) and $\beta$-ratio (Fig. 3) is minor. Most of the difference in the size distributions (Fig. 10, top left) probably comes from the changes in the bulk density. The blowout for grains with iron inclusions is slightly shifted to smaller sizes und the whole distribution is stretched, while in the case of water inclusions the opposite is true. The radial distribution of dust (top right) remains virtually the same.

In terms of thermal emission, the influence of inhomogeneity is very weak, too. Consistently with the modifications in the size distribution, the SED is narrower for ice and slightly broader for iron inclusions. The surface brightness profiles retain their overall shape. Only the $24 \mu \mathrm{m}$ emission in the outer disk becomes slightly stronger and more gently sloping, when iron inclusions are present.

Our conclusion is that inclusions at a $10 \%$ level have only minor effect on the observables. We cannot exclude that more radical changes in the composition would affect the results substantially, but there is currently no observational evidence that would justify such changes.

\subsection{Cratering Collisions}

We turn to an analysis of the underlying collisional model implemented in $A C E$. The detailed physics and outcomes of binary collisions under the conditions of debris disks are poorly known, which represents one of the major sources of uncertainty in our simulation results. Accordingly, in this and subsequent sections, we vary three key parameters that control the treatment of collisions.

We first explore a hypothetical collisional cascade, in which only disruptive collisions operate and the cratering collisions do not occur. This means that we only consider collisions with specific impact energies above the threshold value $\left(Q_{\mathrm{D}}^{\star}\right)$, which shatter both colliders completely. All collisions at lower energies (that would in reality erode one or both of the colliders) are simply ignored.

Thus, an efficient way of eroding larger objects by collisions with much smaller grains is switched off. As a result, grains with $20 \mu \mathrm{m}<s<300 \mu \mathrm{m}$ are more abundant than in the reference model (Fig. 11). And conversely, the number of grains with $s<20 \mu \mathrm{m}$ decreases, so that the maximum of the size distribution is now effectively shifted to about $30 \mu \mathrm{m}$. The explanation is simple. Excluding cratering collisions prolongs the collisional lifetime of larger grains, because smaller impactors that cannot disrupt but would efficiently erode them, now leave them intact (see, e.g., Thébault \& Augereau 2007, and references therein).

The change in the size distribution shifts the SED towards longer wavelengths and slightly narrows it. There is now a lack of emission in the mid and far-IR up to $100 \mu \mathrm{m}$ and an excess emission at sub-mm. The resulting SED clearly violates the data.

Given the deficiency of small particles on highly eccentric orbits, it is not surprising that the optical depth profile becomes very steep. As a consequence, the $70 \mu \mathrm{m}$ brightness profile steepens significantly. In addition, all three profiles fall too low. Like the SED, they are no longer consistent with the observations.

We conclude that cratering collisions cannot be ignored. They seem mandatory to reproduce the observations of the Vega disk with collisional simulations.

\subsection{Energy Threshold for Fragmentation}

In this subsection, we explore the role of the (unknown) tensile strength of the solids, parameterized by the shattering energy $Q_{\mathrm{D}}^{\star}$ in the strength regime. To this end, we decrease $Q_{D, s}$ and $b_{s}$ in Eq. (1) in one simulation ("weak material") and increase them in another one ("hard material").

Figure 11 shows that larger grains benefit from an increase of the energy threshold in a similar way they do from neglecting cratering collisions or from lowering the average impact energies. Their collisional lifetime becomes longer and their amount increases. And conversely, a lower $Q_{\mathrm{D}}^{\star}$ reduces the amount of larger particles. For smaller grains, the number of potentially hazardous impactors is not determined by the change in the critical impactor mass for disruption (that comes along with the change in critical energy) but by the blowout limit, which remains unchanged. Consequently, decreasing $Q_{\mathrm{D}}^{\star}$ "supports" smaller grains, producing a more pronounced first maximum in the size distribution.

In the SED, a lower critical energy leads to a strong shift of the maximum to about $80 \mu \mathrm{m}$, and makes the rise in the mid- to far-IR steeper, whereas the sub-mm and millimeter part lowers and flattens. The opposite changes, albeit less pronounced, are seen for a higher critical energy. In both cases, the agreement between the modeled and observed SED becomes rather worse.

Similarly to the size distribution, the optical depth profile responds to a harder material in nearly the same way as to excluding the cratering collisions. As far as the surface brightness profiles are concerned, the only real improvement can be found in the $160 \mu \mathrm{m}$ profile for larger $Q_{D, s}$ and $b_{s}$. However, this is accompanied with a steepening and flattening of the 70 and $24 \mu \mathrm{m}$ profiles, respectively, which are then clearly inconsistent with the observations.

We conclude that using "weak" or "hard" material with respect to the nominal one does not generally improve agreement with the observations. When improving one of the three surface brightness profiles, for instance, this makes one or two of the others worse. We find, however, that results are very sensitive to the critical energy. Thus moderate modifications in the critical energy can be useful for "fine-tuning" of the models.

\subsection{Fragment Distribution}

One more essential part of the collisional description is the distribution of fragments produced in a single collision. In the reference model we assumed their mass distribution to follow a power-law with an index $\eta=1.833$ retrieved from experiments (Fujiwara et al. 1977), but the experimental conditions do not necessarily reproduce the conditions of debris disks. Here, we try another two disk models, one with an enhanced production of small particles $(\eta=1.95)$ and one with a reduced production $(\eta=1.6)$.

The effect on the size distribution in Fig. 12 is not very strong. An increase of $\eta$ enhances the production of small particles so that the total distribution becomes flatter. This makes the SED broader: the far-IR emission decreases while the sub-mm fluxes are enhanced. Reducing $\eta$, however, trims the production rate of small particles, so that the maximum in the size distribution becomes broader and is shifted to about $15 \mu \mathrm{m}$. Consequently, the SED becomes somewhat narrower, with a steeper rise in the mid-IR, stronger emission in the farIR, and a steeper fall-off in the sub-mm. These changes are minor, so that the SEDs for both $\eta$ values are consistent with 


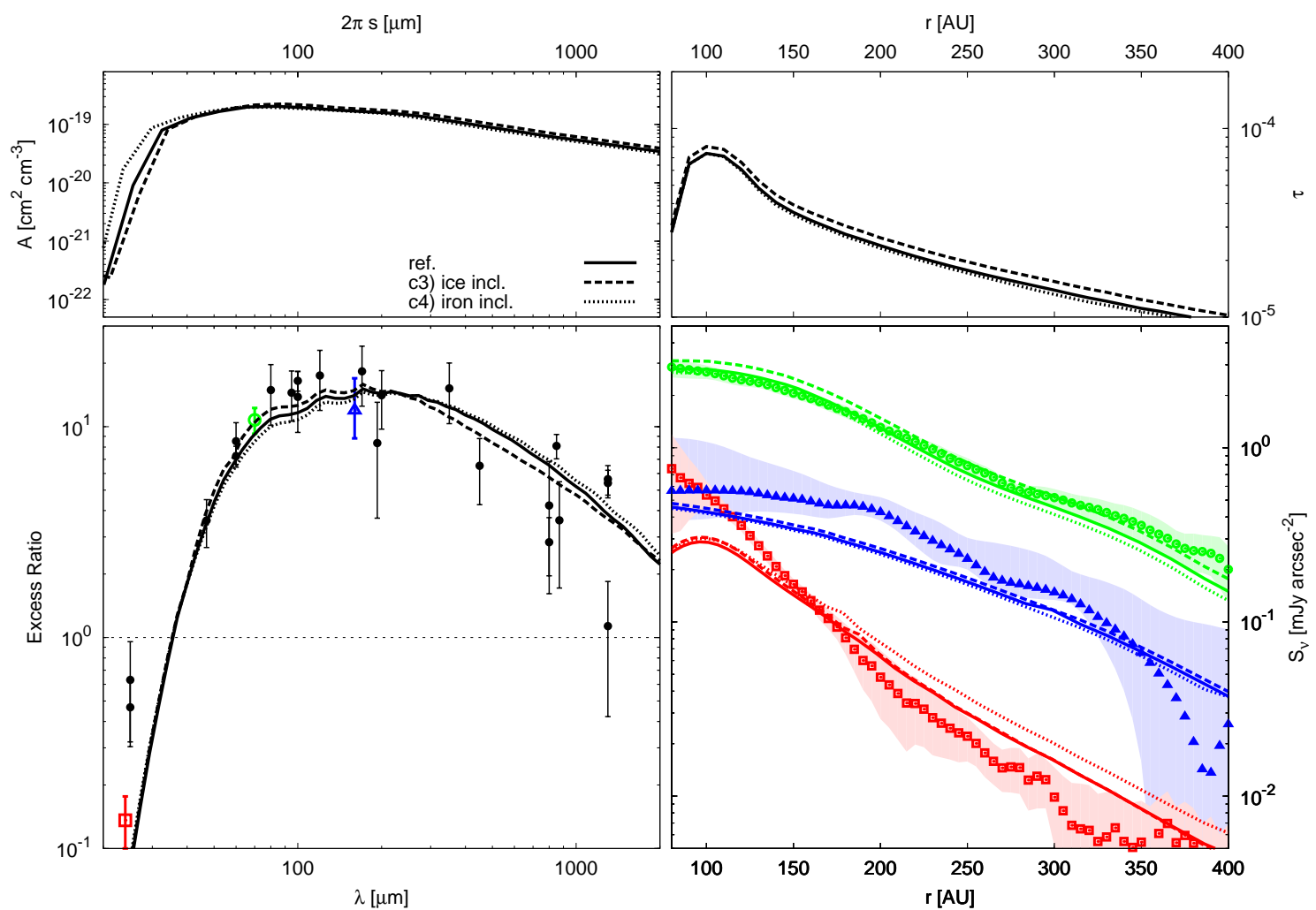

FIG. 10.- Same as Fig. 5 but for disk of particles consisting of an astrosilicate matrix with $10 \%$ water ice (dashed lines) and iron inclusions (dotted lines).

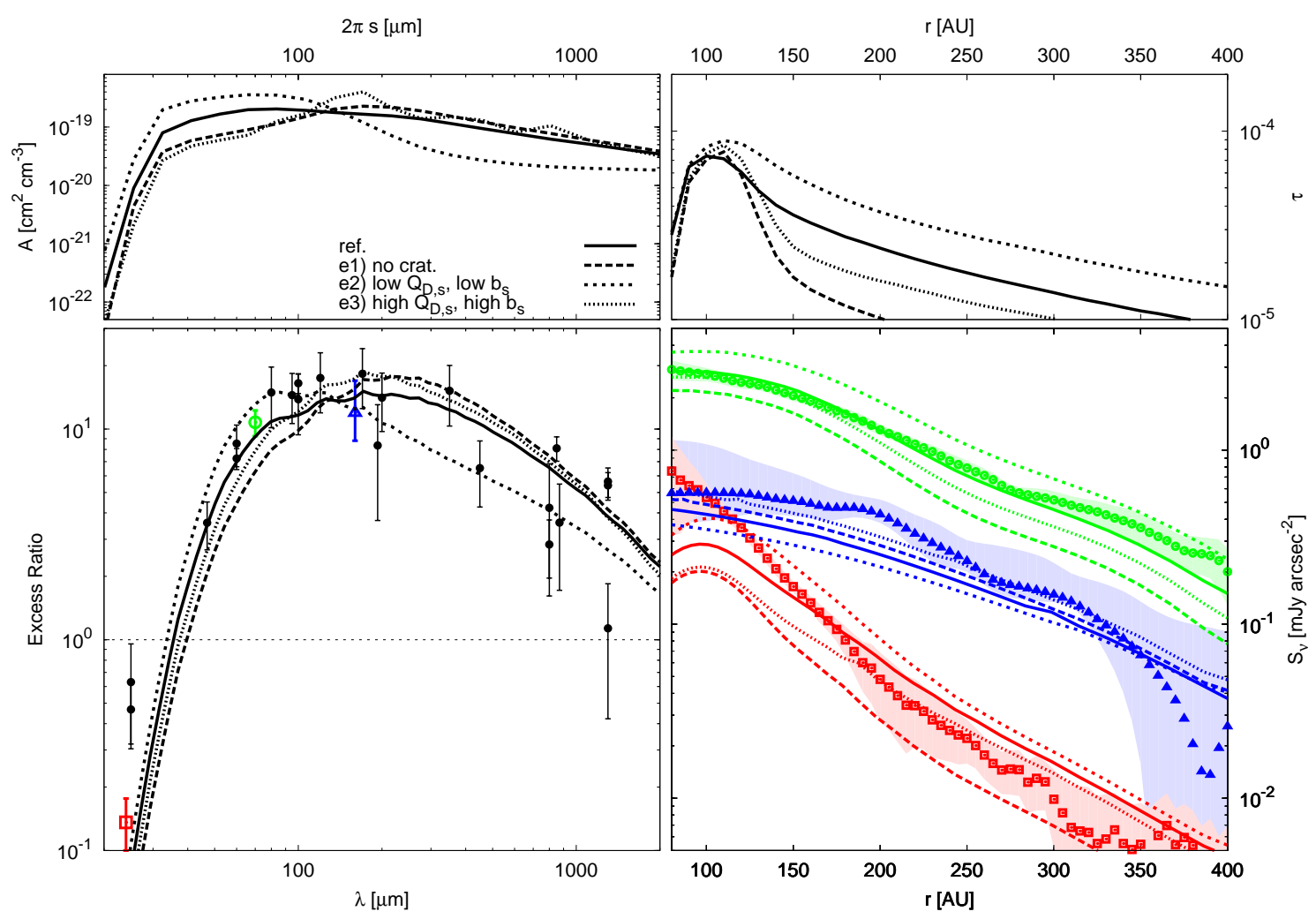

FIG. 11.- Same as Fig. 5 but for a disk without cratering collisions (long dashed) and for a disk material with lower (short dashed) and higher critical shattering energy (dotted). 
the observed SED, as is the SED in the reference model.

Changes in the optical depth are subtle, but not unimportant, particularly at the outer edge of the birth ring. While a lower $\eta$ makes the optical depth profile smoother, a higher $\eta$ creates a slight dip at the outer end of the planetesimal belt. In this radial zone, emission stems predominantly from intermediate-sized grains, which are placed by radiation pressure in moderately eccentric orbits, but still cannot reach the outermost regions of the disk. Reducing $\eta$ increases the amount of these particles compared to the reference model, which smoothens the optical depth profile in this region. And conversely, an increase of $\eta$ depresses the population intermediate-sized grains, causing the dip.

In the radial surface brightness profiles these changes are evident in the $24 \mu \mathrm{m}$ profile, which becomes flatter for small $\eta$ and steeper for high $\eta$ in the region up to about $200 \mathrm{AU}$. The vertical shifts are in agreement with the modifications in the SED.

A conclusion is that a flatter size/mass distribution of fragments with $\eta=1.95$ makes the $24 \mu \mathrm{m}$ brightness profile more consistent with the observed ones, without making other two profiles and the SED worse.

\subsection{The Best Fit}

Different modifications in the previous section have shown no simple way of further improving the agreement of our reference model with the observations. However, we found that variation of some parameters is able to change the results in the desired direction. We now combine several of the modifications that looked promising: the disk was extended inwards, the eccentricities were reduced, the luminosity increased, and a steeper fragment distribution was assumed. Specific parameter values are listed in the last line of Tab. 3.

The result is depicted in Fig. 13 with dashed lines; as always, solid line shows the reference model for comparison. In terms of the SED (bottom left), all photometry data (aside from the IRAS $25 \mu \mathrm{m}$ points) are reproduced within the error bars shortward of $200 \mu \mathrm{m}$. At longer wavelengths, the observational data themselves split into groups that are not in agreement with each other. The model perfectly matches the upper set of points.

In terms of the radial surface brightness profiles, the $160 \mu \mathrm{m}$ profile is nearly the same as in the reference model. The $24 \mu \mathrm{m}$ profile is at about $1 \sigma$ in all regions of the disk. From all three curves the $70 \mu \mathrm{m}$ profile is the closest to the observed one.

\section{DISCUSSION}

\subsection{Modeling the Vega Disk "from the Sources"}

In this paper we have attempted to reproduce observations of the Vega disk by assuming that the observed emission stems from the debris dust, which is produced by a "Kuiper belt" at $\sim 100 \mathrm{AU}$ in a steady-state collisional cascade. To this end, we have performed involved collisional simulations to generate and evolve the disk of solid material from planetesimals to dust and then calculated thermal emission of the dust portion of the disk to confront the results with the available observations. Compared to the commonly used modeling technique, in which dust distributions that provide the best fit to the data are sought, we seek parent planetesimals whose collisional debris could produce emission that matches the observations. This is obviously one major step more than in the traditional method: we try to go back to sources by modeling actual physical processes that operate in a debris disk.

Starting this research, we wanted to test whether our approach can withstand a detailed comparison with various sets of observational data on one particular, and well studied, debris disk system. We have chosen Vega, an archetype debris disk. One particular motivation for this choice was that previous studies uncovered peculiarities, casting doubt on a "standard collisional cascade" as the main mechanism sustaining the disk of Vega.

We understood from the very beginning that our efforts may not be rewarding. The fact that our approach involves a physical modeling to the whole "depth" automatically adds major uncertainties, notably those related to the collisional physics. Contrary to the standard data fitting, we cannot control size and radial distribution of visible dust and we are not at liberty to add, for example, another population of dust to improve agreement with observations. Thus it would be unrealistic to expect that our approach could immediately deliver results that are consistent with observations to that same extent as in the traditional method. It was to our surprise therefore that already with a 'first guess' reference model we are able to reproduce the SED and the modeled mid- to far-IR radial brightness profiles were not inconsistent with Spitzer/MIPS data. We were then able to further improve the agreement by variation of model parameters.

\subsection{Blowout or bound grains?}

It is interesting to trace, why Su et al. (2005) needed an extremely high amount of blowout grains to explain the MIPS observations, whereas we are able to reproduce the same data without any blowout grains (in our models, they make a negligible contribution to the SED and radial brightness profiles at all wavelengths considered and at any distance in the disk). By fitting the MIPS photometry and radial profiles with a single power-law size distribution, Su et al. (2005) found the best fit to be $s_{\min }=1 \mu \mathrm{m}$ and $s_{\max }=50 \mu \mathrm{m}$, with a slope of -3.0 . Assuming nominal stellar luminosity of $L=60 L_{\odot}$, already the compact grains have a blowout limit of $\approx 8 \mu \mathrm{m}$. However, if the grains are highly porous, which of course they may, then the blowout limit will shift to much larger sizes, so that all grains in the $1 \mu \mathrm{m} . . .50 \mu \mathrm{m}$ could indeed be in unbound orbits. An additional argument to favor the radiation pressure-induced outflow seemed to be the deduced brightness profile at $24 \mu \mathrm{m}$ with a slope of $-3 \ldots-4$, since a -3 slope is what blowout particles with nearly constant "terminal" velocities would produce. At that time, it was not yet realized that slopes in the range $-3 \ldots-4$ would equally be typical of an extended disk of small bound grains in elliptic orbits around a parent planetesimal ring, as was found later numerically (Krivov et al. 2006; Thébault \& Augereau 2007) and analytically (Strubbe \& Chiang 2006).

In this study, we assume compact grains and $L=28 L_{\odot}$ in the reference model (note that the very idea to reduce the stellar luminosity would sound strange at the time of the (Su et al. 2005) study, because the fast rotation of Vega was not yet discovered). As a result, the blowout radius reduces to $\approx 4 \mu \mathrm{m}$. Thus where Su et al. had $1 \mu \mathrm{m} . . .50 \mu \mathrm{m}$ grains, we have $4 \mu \mathrm{m} . . .50 \mu \mathrm{m}$ ones. For a -3.0 size distribution slope, the emitting cross section area is equally distributed over the sizes. Thus, even if we take into account that smaller grains are somewhat hotter than larger ones, by excluding the $1 \mu \mathrm{m} . . .4 \mu \mathrm{m}$ subrange we do not lose much of the $24 \mu \mathrm{m}$ emission compared to Su et al. The radial distribution of dust in our model, as explained above, is not very different either. 


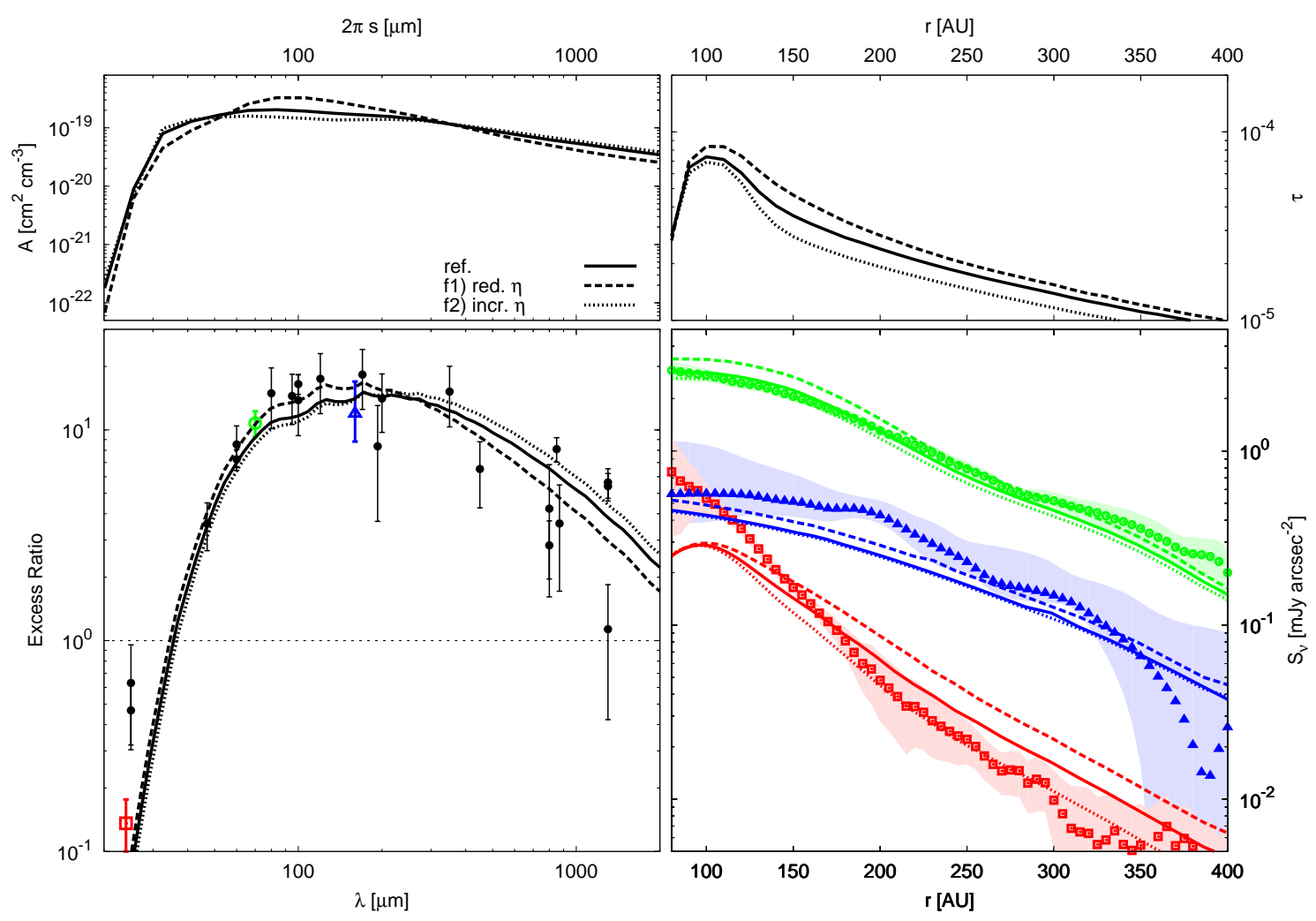

FIG. 12.- Same as Fig. 5 but for model disks with a flatter (dashed) and steeper distribution of fragments (dotted).

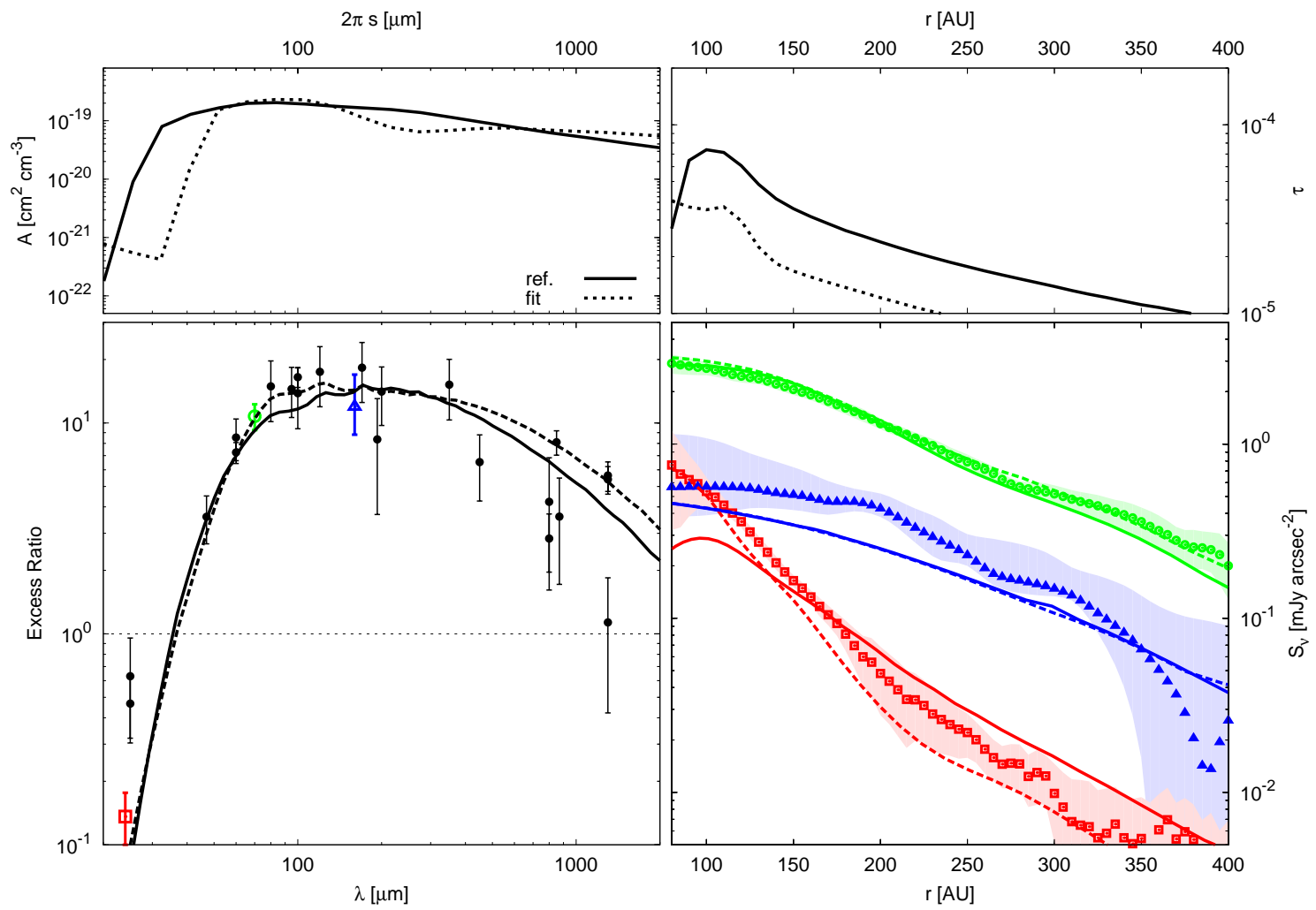

FIG. 13. - Same as Fig. 5 but for our best-fit model (dashed lines). 
That is why we arrive at a similar level of $24 \mu \mathrm{m}$ emission. In terms of dust mass, the difference is even smaller. Indeed, the dust masses we derive here $\left(6.6 \times 10^{-3} M_{\oplus}\right.$ in the reference model, see Tab. (4) are close to those derived by $\mathrm{Su}$ et al. $\left(2.8 \times 10^{-3} M_{\oplus}\right)$.

\subsection{Mass loss from the disk}

We now make estimates of the mass loss from the disk. The total mass of dust (up to $1 \mathrm{~mm}$ ) in the reference model is $\sim 7 \times 10^{-3} M_{\oplus}$ (Table 3). Assuming for simplicity that the size distribution follows a -3.5 power law, the mass of the smallest bound grains (say, up to $10 \mu \mathrm{m}$ ) is $\sim 7 \times 10^{-3} M_{\oplus} \times$ $\sqrt{10 \mu \mathrm{m} / 1 \mathrm{~mm}} \sim 7 \times 10^{-4} M_{\oplus}$. The steady-state mass of blowout grains is then by a factor of 100 smaller (a strength of the dip in the size distribution, which is the ratio of the collisional lifetime of bound grains to the disk-crossing time of unbound ones, see Fig. 4 right), giving $\sim 7 \times 10^{-6} M_{\oplus}$. Their lifetime is $\sim 1000 \mathrm{yr}$, so that the mass loss rate is $\sim 7 \times 10^{-9} M_{\oplus} \mathrm{yr}^{-1}$. Therefore, over the system's age, $350 \mathrm{Myr}$, the disk must have lost $\sim 2 M_{\oplus}$ of material. This estimate is consistent with the difference between the initial and final disk masses given in Tab. 4 typically a few $M_{\oplus}$.

\subsection{The $24 \mu \mathrm{m}$ Emission}

It was "the $24 \mu \mathrm{m}$ problem" - an apparently too strong and radially extended $24 \mu \mathrm{m}$ emission compared to what was expected from dust in bound orbits - that triggered debate on whether the Vega disk contains an excessive number of small blowout grains, incompatible with a steady-state collisional cascade (Su et al. 2005). Thus we now discuss in more detail how the $24 \mu \mathrm{m}$ flux predicted by our models compares to Spitzer data.

Although the models presented here are in a reasonable agreement with observations, most of them somewhat underestimate the observed $24 \mu \mathrm{m}$ emission in the parent ring region, at $80-120 \mathrm{AU}$ or $10^{\prime \prime}-15^{\prime \prime}$, while slightly overestimating it farther out from the star. As a result, the total flux outside $10^{\prime \prime}$, which is dominated by the flux from the ring region, is slightly below the observed value. For example, our reference model predicts $0.40 \mathrm{Jy}$ outside $10^{\prime \prime}$, while the observed flux is $0.53 \mathrm{Jy}$. These deviations are subtle and probably not of serious concern. We argue that they may simply be caused by the roughness of the model (see Sect.6.7). Indeed, we were able to find a combination of model parameters (Sect. 5.9) which reproduced the observed $24 \mu \mathrm{m}$ profile outside $10^{\prime \prime}$ quite well.

However, the question of $24 \mu \mathrm{m}$ emission from the inner system $\left(<10^{\prime \prime}\right)$ remains open. Our analysis in Sect. 2] yields a total $24 \mu \mathrm{m}$ flux from $4^{\prime \prime}$ (30 AU) outward of $0.94 \mathrm{Jy}$, although the true value may be lower, because central part of the MIPS images is saturated. Assuming, however, the data to be accurate, the rise of the $24 \mu \mathrm{m}$ flux from $10^{\prime \prime}$ inward can hardly be explained with the models presented here. A natural explanation would be an additional dusty belt in the system at $\sim 10$ AU. Such a belt could enhance the $24 \mu \mathrm{m}$ emission coming from the "main" disk. As yet it is not clear, however, if any constraints on such a belt can be found in the Spitzer/IRS spectrum of Vega. Nor is it clear whether the inner system may accommodate such a belt if, as conjectured, it hosts one or more close-in planets. In the future, this simple hypothesis could be checked or falsified directly, for instance with mid-IR interferometry.

\subsection{The $850 \mu \mathrm{m}$ Emission}

So far, we confined our analysis to resolved images in the IR and did not consider explicitly sub-mm and radio images. The main reason for that is a low resolution of such measurements, implying that only weak constraints can be put on the radial brightness profiles at long wavelengths. However, at least the total flux at sub-mm wavelengths derived from the images serves as an additional test to the models.

In an analysis by Su et al. (2005), the observed sub-mm emission could not be reproduced with a two-component dust disk ( $2 \mu \mathrm{m}$ and $18 \mu \mathrm{m}$ in radius) that was sufficient to fit all available data at shorter wavelengths. To cope with the problem, they artificially added a population of larger grains, with a radius of $215 \mu \mathrm{m}$. In our approach, solids from dust to planetesimals have a continuous size distribution, which is not postulated, but physically modeled. From Figs. 54 13 (left bottom panels) it is apparent that our simulations naturally reproduce the sub-mm flux with a reasonable accuracy. An additional consistency check that we made was to calculate the $850 \mu \mathrm{m}$ profile of our best fit model (Sect. 5.9 and convolve it with a Gaussian of $16^{\prime \prime}$ beam size. The resulting profile was then compared with the SCUBA profile extracted by $\mathrm{Su}$ et al. (2005) from the original images published by (Holland et al. 1998). The modeled profile is slightly narrower than that observed and the maximum between 50 and $100 \mathrm{AU}$ is by a factor of two lower. Given the large width of the PSF and calibration uncertainties of $S C U B A$ observations, and that Mie calculations likely underestimate sub-mm emission (as discussed in Sect. 6.7), we deem the agreement with the data satisfactory.

\subsection{Model Parameters}

As we saw, even our "best fit" (Fig. 13) cannot match the observations perfectly. The discrepancies could stem either from the fact that our "first-guess" choice of parameters in the reference model was not the best, from the limitations or shortcomings of the collisional and thermal emission model, or for the two reasons together. In this and the next section, we address the both possible reasons in turn.

In Sect. 5, we investigated in detail how the dust distributions and thermal emission are affected by a large array of physical parameters:

- The "collisional" age of the disk, i.e. the time elapsed from the onset of the steady-state collisional evolution

- The location and extension of the parent planetesimal belt

- The dynamical excitation of the belt, parameterized by the maximum orbital eccentricity of planetesimals

- The stellar luminosity

- The chemical composition of the visible dust

- Several parameters that control individual collisions between the objects in the disk, from planetesimal to dust. In particular, we checked the role of cratering collisions, the critical impact energy threshold for disruptive collisions, and the distribution of fragments after a collision.

We have identified two parameters that have a major influence. One is the stellar luminosity, and its effect is particularly interesting. One might think that a more luminous central star 
would increase dust temperatures, but this is untrue. A higher luminosity implies a larger blowout size. Thus the most abundant grains in the disk - those just above the blowout limit are now larger and therefore colder (Fig. 4, top left). The net result is that, counterintuitively, the characteristic dust temperature in the disk does not change much (in the outer disk it even decreases) with increasing luminosity of the central star. Another parameter is the efficiency of cratering collisions. In the extreme case where these are switched off, the simulation results contradict the observations.

Contrary to our expectations, it turned out that other parameters probed have only minor to moderate effect on the SED and surface brightness distribution of the disk, with consequences being two-fold. On the one hand, this implies that the model predictions are rather robust. As an example, our reference model was already in a rough agreement with observations, so that further improvements through "parameter tuning" seemed easy, but these turned out to be difficult. We find meaningful parameter sets that provide better fit to observations than the reference model does (see Fig. 13), but the agreement is somewhat worse than in the study of Su et al. (2005). On the other hand, a weak dependence of the observables on many parameters restricts the possibility of constraining them, which is somewhat unlucky, because placing constraints on model parameters is one of the important goals of the modeling.

\subsection{Limitations of the Model}

We are fully aware that our modeling approach, as every other, involves a number of simplifying assumptions that may limit its applicability and influence the results. Here, we list the most important caveats.

Many assumptions have been made in describing collisional physics. Our collisional prescription approximates the critical shattering energy with two power laws (Eq. 1), which may be particularly crude at dust sizes (e.g. Thébault \& Augereau 2007). The mass of the largest fragment and the distribution of smaller debris may deviate from what was assumed here, and any real disk should be composed of objects whose mechanical properties (and even the bulk density) vary from one object to another (e.g. pre-shattered objects could be less dense and more loosely bound than pristine ones).

A major simplifying assumption in treating the dynamics of planetesimals and their dust is that we ignore alleged planetary perturbers interior to the main belt. The consequences are discussed in Sect. 6.9. At dust sizes, we did not take into account P-R drag, its role is discussed in Sect.6.8 In calculating the radiation pressure force acting on dust grains, we assumed them to be compact and spherical, thus ignoring possible nonradial effects (e.g. Kimura et al.2002). Like mechanical properties, optical properties of dust may vary from one grain to another, resulting in different response to radiation pressure, different temperatures, and different thermal fluxes even for like-sized particles (Krivov et al. 2006). Furthermore, even spherical particles are treated in an approximate way. We applied Mie theory to model the emission properties. Although this method is classical and commonly used, it should be treated with caution. One particular concern is that Mie calculations probably underestimate the emission in the sub$\mathrm{mm}$ and radio due to neglected size effects (Stognienko et al. 1995; Krivov et al. 2008).

\subsection{The Role of the Poynting-Robertson Effect}

Poynting-Roberston (P-R) drag mostly affects smallest particles and thus emission at shortest wavelengths considered. The P-R force moves such grains inward, placing some of them interior to the inner edge of the birth ring. The warm emission of these particles especially around the inner edge of the birth ring should increase.

However, with the Vega disk's relatively high optical depth $\left(8.3 \times 10^{-4}\right.$ at $100 \mathrm{AU}$ in the reference model $)$, the collisional timescales of dust grains are shorter than timescales over which Poyining-Roberston (P-R) drag causes their appreciable radial displacement (Fig. 2). Thus the Vega disk can be referred to as a collision-dominated, rather then transportdominated disk (Krivov et al. 2000; Wyatt 2005a). Still, it is useful to check to what extent PR drag may affect the results in terms of SED and brightness profiles.

To this end, we have switched on P-R drag in our reference model. Unfortunately, when a drag force, the P-R force in our case, is added to a collisional model, the mass-time scaling law (Sect. 3.1) is no longer valid. One has to take the initial disk mass that yields a disk with the "correct" dust mass after $350 \mathrm{Myr}$, i.e. the dust mass that gives the maximum of the SED at the level actually observed. This means trial and error, i.e. several $A C E$ runs with different initial disk masses followed by SEDUCE and SUBITO runs. What makes the modeling even more demanding, is that the presence of a drag force implies diffusion in the phase space of pericenters and eccentricities, which slows down each $A C E$ run appreciably. We had to perform four $A C E$ runs, each of which took about 20 core-days CPU time.

The "right" dust mass after 350 Myr of evolution with P-R was achieved when the initial disk mass was set to $20.5 M_{\oplus}$ (instead of $18.9 M_{\oplus}$ in the reference model without P-R), and the final disk mass was $18.0 M_{\oplus}$ (instead of $16.3 M_{\oplus}$ without $\mathrm{P}-\mathrm{R})$. As expected, the influence of P-R on the $160 \mu \mathrm{m}$ and $70 \mu \mathrm{m}$ turned out to be completely negligible. At $24 \mu \mathrm{m}$, the emission in the outer disk increases by $\sim 10 \%$ and in the birth ring (at $80 \mathrm{AU}$ ) by $\sim 60 \%$. Thus the whole $24 \mu \mathrm{m}$ profile gets somewhat steeper, and agrees with observations slightly better than the original profile in the reference model (solid lines in Figs. 513). However, the improvement is only minor, and we conclude that the P-R effect can safely be neglected in modeling the Vega system.

\subsection{Presumed Planets in the Vega System}

One major caveat not discussed in Sect.6.7 is that our collisional model, implemented in the $A C E$ code, ignores effects of a possible planet (or planets) interior to the planetesimal belt. Below we briefly outline the facts that point to the presence of such planets in the Vega system and discuss how these perturbers may affect the observed properties of the debris disk.

Asymmetries in the Vega disk were first discovered by Holland et al. (1998) in a SCUBA $850 \mu \mathrm{m}$ image, and subsequent sub-mm and radio observations have confirmed a clumpy ring structure. Wilner et al. (2002) introduced the idea of a Jupiter mass planet trapping dust in mean-motion resonances. They applied $N$-body simulations and thermal emission calculations to model this scenario and achieved a reasonable agreement with their IRAM map. An in-depth investigation on the Vega system dynamics was performed by Wyatt (2003) who suggested that a Neptune-mass planet, migrating outward from 40 to 65 AU over a time span of $\sim 56$ Myr, may have cleared the inner part of the assumed planetesimal disk and trapped a significant amount of material in the $3: 2$ and $2: 1$ resonances, thus creating two 
clumps as seen by Holland et al. (1998). Later on, Reche et al. (2008) generalized this theory to account for eccentric planetary orbits. Their findings are similar to those of Wyatt (2003) with the difference that they require a Saturn-mass planet on a low eccentricity orbit to account for the brightness asymmetry. For the clumps to be visible against the non-resonant background, low planetesimal eccentricities of $<0.1$ are necessary. Planets with masses greater than $\approx 2 M_{\text {Jupiter }}$ would raise planetesimal eccentricities to about 0.2. Besides, too massive planets would quickly deplete the disk.

As shown in section 5.3, our simulations slightly favor low planetesimal eccentricities up to 0.1. This is in agreement with the limit given by Reche et al. (2008). Still, a question arises whether non-inclusion of the azimuthal structure in our simulations ( $A C E$ treats rotationally-symmetric disks) is a reasonable assumption. Indeed, Wyatt (2006) investigated the dust production in a clumpy disk of resonant planetesimals and showed that local dust production from the clumps is strongly enhanced and conversely, it is depressed between the clumps. We argue, however, that the net effect on the SED and radial profiles of brightness is much weaker, because these depend on the azimuthally-averaged dust production rates. Queck et al. (2007) found that the average collisional rate in a resonant planetesimal belt is typically not more than twice as high as in a similar non-resonant belt, while the average collisional velocities are nearly unaffected by the resonant clumping. Thus a collisional cascade in a resonant, clumpy belt can be approximated by a cascade in a non-resonant, rotationally-symmetric belt with the same mass at the same location, but somewhat higher orbital eccentricities of planetesimals to mimic moderately enhanced collisional rates. We stress that this is only valid when considering azimuthally-averaged observables, not the azimuthallyresolved structure seen in the images. For example, our approach is not suitable to make predictions for spiral structure expected to emanate from the clumps. Wyatt (2006) argues that such structure should be seen in mid- to far-IR images, and that it is not, may simply be due to insufficient resolution of the Spitzer/MIPS images or confusion in the photospheric subtraction.

Throughout the study, we assumed that the initial eccentricities and inclinations of the parent bodies in the planetesimal belt are distributed according to energy equipartition. This assumption would be reasonable if the distribution of orbits was controlled by mutual collisions and gravitational scattering among planetesimals, but it may not hold as soon as resonant interaction with planets occurs. A well-known example is our Edgeworth-Kuiper belt, in which the eccentricities and inclinations of objects are distributed differently (e.g. Brown 2001).

Apart from the suspected planet that sculpts the main belt, the Vega system may contain more planets closer in. In fact, a damped outward migration of the presumed planet that explains the clumps requires the presence of another, more massive planet in the system closer in (e.g. Gomes et al. 2004). An inner planet, or planets, could stir the disk (Mustill \& Wyatt 2009). Furthermore, several planets together could produce intricate combined dynamical effects on the main planetesimal belt and its dust. However, it seems premature to discuss them until new observations have delivered evidence for these planets.

\subsection{The Exozodi in the Vega System}

As mentioned in the introduction, dust was surprisingly discovered in the innermost part of the Vega system, inside 1 AU (Absil et al. 2006). Although reminiscent of the zodiacal cloud of the solar system, this "exozodi" of Vega remains a mystery. It seems to be far too dusty, and the grain sizes retrieved from observations far too small, to be explained by collisions in an "asteroid belt" or evaporation of comets. One possibility would be a transport of planetesimals from the "main" debris disk inward and their subsequent disruption or evaporation. Such a transport would require the presence of at least two planets. In fact, a two-planet configuration - a "Jupiter" inside and a "Saturn" outside that shapes the main disk - could suffice (Vandeportal et al., in prep.). Thus, the very existence of the exozodi may strengthen the expectation that Vega hosts several planets, as discussed above.

The direct contribution of the exozodi to the emission at $24 \mu \mathrm{m}$ amounts to $\approx 0.6 \mathrm{Jy}$. This is about the emission which is lacking provided the used photometry data is accurate. However, as the exozodi could not be resolved with Spitzer and the image is saturated at the stellar position, this very inner part of the Vega disk cannot have affected the observation in the outer parts of the system and can therefore be considered negligible. Still, if not directly, the Vega exozodi could have an indirect impact on the measured dust emission. Dust inside 1 AU could have a shielding effect on dust located farther out. However, a simple estimate shows that the amount of stellar radiation to which outer dust is exposed would only reduce by a factor of $\sim 10^{-5}$. Thus, we conclude that the very inner part of the system has no impact on the outer disk's emission analyzed in this paper.

\section{CONCLUSIONS}

Our analysis suggests that the debris disk of Vega is compatible with a standard scenario, in which visible dust originates from a steady-state collisional cascade operating in a "Kuiper belt", whose existence at 100 AU from the star is evident in sub-mm images. We model the dust production from the sources, and find that thermal emission of the resulting dust is fully consistent with the photometric data across the entire wavelength range from mid-IR to radio covered by observations. Furthermore, we are able to naturally reproduce the radial brightness profiles at 24,70 , and $160 \mu \mathrm{m}$ derived from Spitzer/MIPS observations. Finally, the modeled emission agrees with the low-resolution images at $850 \mu \mathrm{m}$ taken with $S C U B A$.

If the Vega disk is maintained by a steady-state collisional cascade, which appears likely, its total mass (in $\lesssim 100 \mathrm{~km}$ sized bodies) must fall in the range from several to several tens of Earth masses. Provided that collisional cascade has been operating over much of the Vega age of $350 \mathrm{Myr}$, the disk must have lost a few Earth masses of solids during that time. Further constraints of the parameters of the system and physical processes operating in the disk are as follows. A reasonable amount of stirring should be present in the planetesimal ring. We demonstrate that planetesimals are likely to have eccentricities of the order of $\approx 0.1 \ldots 0.3$, but the origin of stirring cannot be constrained. It may come, for instance, from a presumed giant planet interior to the belt, or the disk can be self-stirred by largest, Pluto-sized planetesimals. Next, we show that the modeling results sensitively depend on the luminosity of the central star. Importantly, in the particular case of Vega, using a reduced radiation flux from the stellar surface at low latitudes, which was derived from its fast rotation, is mandatory to match modeled dust emission to the 
data. Another important prerequisite for this is to include cratering collisions between the disk solids as a collisional outcome into the model.

Another goal of this study was to use the Vega disk as a stringent test for our modeling approach, which is a physical modeling of a debris disk "from sources" (Krivov et al. 2008). We deem the test successful. We have shown that the approach does work and has a potential to deliver constraints, most notably on the properties of directly invisible planetesimals, that are not possible to put with other methods. Our collisional and thermal emission model needs to be further tested and "calibrated" on other resolved debris disk systems. Then, it can be used as a routine procedure in dynamical modeling of debris disks, both known and expected to be detected with facilities like Herschel or ALMA.

The data reduction and modeling presented here describe and explain the outer system, outside $\approx 80 \mathrm{AU}$. Inside that distance, our analysis of $24 \mu \mathrm{m}$ emission observed by Spitzer/MIPS indicates a possible rise of the $24 \mu \mathrm{m}$ flux from $10^{\prime \prime}$ inward, although it is not certain, because central part of the MIPS $24 \mu \mathrm{m}$ images is saturated. Assuming, however, the data to be accurate, they cannot be explained by the models presented here. A natural explanation would be an additional dusty belt in the system at $\sim 10$ AU. Such a belt could enhance the $24 \mu \mathrm{m}$ emission coming from the "main" disk. In the future, this hypothesis could be checked or falsified directly, for instance with a more accurate mid-IR photometry of mid-IR interferometry. On any account, further observational and theoretical effort is required to shed more light onto the inner part of the Vega system, including alleged planets, possible planetesimal belts and dust rings, of which one - an "exozodi" at just $\sim 1 \mathrm{AU}$ - has recently been discovered with near-IR interferometry. It is the inner system that must eventually bring clues to the entire architecture and the formation history of the Vega system. A better knowledge of the inner part of the system, most notably suspected planets there, will also result in a better understanding of how the outer debris disk operates.

We thank Olivier Absil, Jean-Charles Augereau, Hervé Beust, Hiroshi Kimura, Harald Mutschke, Kate Su, Phillippe Thébault, and Mark Wyatt for numerous fruitful discussions on various aspects of this work. The review by the anonymous referee greatly helped to improve the paper. Part of this work was supported by the German Deutsche Forschungsgemeinschaft, DFG projects $\mathrm{Kr} 2164 / 5-1$ and $\mathrm{Kr} 2164 / 8$ 1, by the Deutscher Akademischer Austauschdienst (DAAD), project D/0707543, and by the International Space Science Institute in Bern ("Exozodiacal Dust Disks and Darwin" working group ${ }^{3}$ ). SM is funded by the Graduate Student Fellowship of the Thuringia State. AK and TL are grateful to the Isaac Newton Institute for Mathematical Sciences in Cambridge where the final stages of this work were carried out in the framework of the program "Dynamics of Discs and Planets".

\footnotetext{
${ }^{3}$ http://www.issibern.ch/teams/exodust/
} 


\section{REFERENCES}

Absil, O., et al. 2006, A\&A, 452, 237

Aufdenberg, J. P., et al. 2006, ApJ, 645, 664

Augereau, J., \& Beust, H. 2006, A\&A, 455, 987

Aumann, H. H., et al. 1984, ApJ, 278, L23

Beichman, C. A., et al. 2005, ApJ, 626, 1061

Benz, W., \& Asphaug, E. 1999, Icarus, 142, 5

Brown, M. E. 2001, AJ, 121, 2804

Burns, J. A, Lamy, P. L., \& Soter, S. 1979, Icarus, 40, 1

Chen, C. H., Fitzgerald, M. P., \& Smith, P. S. 2008, ApJ, 689, 539

Chen, C. H., et al. 2006, ApJS, 166, 351

Chini, R., Kruegel, E., \& Kreysa, E. 1990, A\&A, 227, L5

Davis, D. R., Chapman, C. R., Weidenschilling, S. J., \& Greenberg, R. 1985 Icarus, 62,30

Dent, W. R. F., Walker, H. J., Holland, W. S., \& Greaves, J. S. 2000, MNRAS, 314, 702

Durda, D. D., Bottke, W. F., Nesvorný, D., Enke, B. L., Merline, W. J., Asphaug, E., \& Richardson, D. C. 2007, Icarus, 186, 498

Durda, D. D., \& Dermott, S. F. 1997, Icarus, 130, 140

Durda, D. D., Greenberg, R., \& Jedicke, R. 1998, Icarus, 135, 431

Fujiwara, A., Kamimoto, G., \& Tsukamoto, A. 1977, Icarus, 31, 277

Gomes, R. S., Morbidelli, A., \& Levison, H. F. 2004, Icarus, 170, 492

Harper, D. A., Loewenstein, R. F., \& Davidson, J. A. 1984, ApJ, 285, 808

Harvey, P. M., Wilking, B. A., \& Joy, M. 1984, Nature, 307, 441

Hauschildt, P. H., Allard, F., \& Baron, E. 1999, ApJ, 512, 377

Heinrichsen, I., Walker, H. J., \& Klaas, U. 1998, MNRAS, 293, L78

Holland, W. S., et al. 1998, Nature, 392, 788

Holsapple, K. A. 1994, Planet. Space Sci., 42, 1067

Jura, M., et al. 2004, ApJS, 154, 453

Kenyon, S. J., \& Bromley, B. C. 2004a, AJ, 127, 513

-. 2004b, AJ, 128, 1916

-. 2008, ApJS, 179, 451

Kimura, H., Okamoto, H., \& Mukai, T. 2002, Icarus, 157, 349

Koerner, D. W., Sargent, A. I., \& Ostroff, N. A. 2001, ApJ, 560, L181

Kolokolova, L., \& Gustafson, B. A. S. 2001, Journal of Quantitative Spectroscopy and Radiative Transfer, 70,611

Krivov, A. V., Löhne, T., \& Sremčević, M. 2006, A\&A, 455, 509

Krivov, A. V., Mann, I., \& Krivova, N. A. 2000, A\&A, 362, 1127

Krivov, A. V., Müller, S., Löhne, T., \& Mutschke, H. 2008, ApJ, 687, 608

Krivov, A. V., Queck, M., Löhne, T., \& Sremčević, M. 2007, A\&A, 462, 199

Krivov, A. V., Sremčević, M., \& Spahn, F. 2005, Icarus, 174, 105

Laor, A., \& Draine, B. T. 1993, ApJ, 402, 441

Lisse, C. M., Beichman, C. A., Bryden, G., \& Wyatt, M. C. 2007, ApJ, 658, 584
Lisse, C. M., Chen, C. H., Wyatt, M. C., \& Morlok, A. 2008, ApJ, 673, 1106

Löhne, T., Krivov, A. V., \& Rodmann, J. 2008, ApJ, 673, 1123

Lynch, D. W., \& Hunter, W. R. 1991, in Handbook of Optical Constants of Solids II, ed. E. D. Palik (San Diego, CA: Academic Press), 385

Makarov, V. V., Gaume, R. A., \& Andrievsky, S. M. 2005, MNRAS, 362, 1109

Marsh, K. A., Dowell, C. D., Velusamy, T., Grogan, K., \& Beichman, C. A. 2006, ApJ, 646, L77

Mustill, A., \& Wyatt, M. 2009, ArXiv e-prints

Paolicchi, P., Verlicchi, A., \& Cellino, A. 1996, Icarus, 121, 126

Peterson, D. M., et al. 2006, Nature, 440, 896

Queck, M., Krivov, A. V., Sremčević, M., \& Thébault, P. 2007, Cel. Mech. Dyn. Astron., 99, 169

Reche, R., Beust, H., Augereau, J.-C., \& Absil, O. 2008, A\&A, 480, 551

Schütz, O., Meeus, G., \& Sterzik, M. F. 2005, A\&A, 431, 175

Stapelfeldt, K. R., et al. 2004, ApJS, 154, 458

Stognienko, R., Henning, T., \& Ossenkopf, V. 1995, A\&A, 296, 797

Strubbe, L. E., \& Chiang, E. I. 2006, ApJ, 648, 652

Su, K. Y. L., et al. 2005, ApJ, 628, 487

-. 2006, ApJ, 653, 675

Takagi, Y., Mizutani, H., \& Kawakami, S.-I. 1984, Icarus, 59, 462

Thébault, P., \& Augereau, J.-C. 2007, A\&A, 472, 169

Thébault, P., Augereau, J. C., \& Beust, H. 2003, A\&A, 408, 775

Thébault, P., \& Wu, Y. 2008, A\&A, 481, 713

Thébault, P., Augereau, J.-C., \& Beust, H. 2003, A\&A, 408, 775

Walker, H. J., \& Wolstencroft, R. D. 1988, PASP, 100, 1509

Warren, S. G. 1984, Appl. Opt., 23, 1206

Wilner, D. J., Holman, M. J., Kuchner, M. J., \& Ho, P. T. P. 2002, ApJ, 569 , L115

Wyatt, M. C. 2003, ApJ, 598, 1321

Wyatt, M. C. 2005a, A\&A, 433, 1007

-. 2005b, A\&A, 440, 937

-. 2006, ApJ, 639, 1153

-. 2008, ARA\&A, 46, 339

Wyatt, M. C., Smith, R., Greaves, J. S., Beichman, C. A., Bryden, G., \& Lisse, C. M. 2007a, ApJ, 658, 569

Wyatt, M. C., Smith, R., Su, K. Y. L., Rieke, G. H., Greaves, J. S.,

Beichman, C. A., \& Bryden, G. 2007b, ApJ, 663, 365

Zuckerman, B., \& Becklin, E. E. 1993, ApJ, 414, 793 